\title{
Bayesian joint muographic and gravimetric inversion applied to volcanoes
}

\author{
Anne Barnoud ${ }^{\oplus},{ }^{1,2}$ Valérie Cayol, ${ }^{2,3}$ Valentin Niess, ${ }^{1}$ Cristina Cârloganu, ${ }^{1}$ \\ Peter Lelièvre, ${ }^{4}$ Philippe Labazuy ${ }^{2}$ and Eve Le Ménédeu ${ }^{1}$ \\ ${ }^{1}$ Université Clermont Auvergne, CNRS/IN2P3, LPC, F-63000 Clermont-Ferrand, France.E-mail: anne.barnoud@gmail.com \\ ${ }^{2}$ Université Clermont Auvergne, CNRS, IRD, OPGC, Laboratoire Magmas et Volcans, F-63000 Clermont-Ferrand, France \\ ${ }^{3}$ Université de Lyon, Laboratoire Magmas et Volcans, UJM-UCA-CNRS-IRD, F-42023 Saint-Étienne, France \\ ${ }^{4}$ Memorial University of Newfoundland, St. Johns, Newfoundland and Labrador, A1B 3X5, Canada
}

Received 2019 June 26; in original form 2018 December 12

\begin{abstract}
SUMMAR Y
Gravimetry is a technique widely used to image the structure of the Earth. However, inversions are ill-posed and the imaging power of the technique rapidly decreases with depth. To overcome this limitation, muography, a new imaging technique relying on high energy atmospheric muons, has recently been developed. Because muography only provides integrated densities above the detector from a limited number of observation points, inversions are also ill-posed. Previous studies have shown that joint muographic and gravimetric inversions better reconstruct the 3-D density structure of volcanic edifices than independent density inversions. These studies address the ill-posedness of the joint problem by regularizing the solution with respect to a prior density model. However, the obtained solutions depend on some hyperparameters, which are either determined relative to a single test case or rely on ad-hoc parameters. This can lead to inaccurate retrieved models, sometimes associated with artefacts linked to the muon data acquisition. In this study, we use a synthetic example based on the Puy de Dôme volcano to determine a robust method to obtain the resulting model closest to the synthetic model and devoid of acquisition artefacts. We choose a Bayesian approach to include an $a$ priori density model and a smoothing by a Gaussian spatial correlation function relying on two hyperparameters: an a priori density standard deviation and an isotropic spatial correlation length. This approach has the advantage to provide a posteriori standard deviations on the resulting densities. Using our synthetic volcano, we investigate the most reliable criterion to determine the hyperparameters. Our results suggest that $k$-fold Cross-Validation Sum of Squares and the Leave One Out methods are more robust criteria than the classically used L-curves. The determined hyperparameters allow to overcome the artefacts linked to the data acquisition geometry, even when only a limited number of muon telescopes is available. We also illustrate the behaviour of the inversion in case of offsets in the a priori density or in the data and show that they lead to recognizable structures that help identify them.
\end{abstract}

Key words: Gravity anomalies and Earth structure; Joint inversion; Tomography.

\section{INTRODUCTION}

The 3-D density structure of volcanic edifices is classically inferred from the inversion of gravimetric data (e.g. Camacho et al. 1997; Cella et al. 2007; Linde et al. 2014). Gravimetry provides measurements of the gravity field at multiple locations throughout the study area, corresponding to the integrated effect of the whole Earth and sensitive to the local density variations. The inversion of gravimetric data is a non-unique process, requiring strong a priori geological information to constrain the models or a combination with other geophysical data such as seismic traveltimes (e.g. Onizawa et al. 2002; Coutant et al. 2012).

Muography is a method that emerged from the field of particle physics. Using atmospheric muons, which result from the interaction of cosmic rays with the atmosphere, muography provides 2-D images of integrated densities. Muons are charged leptons, alike 
to electrons, but $\sim 200$ times heavier. They interact with matter through various stochastic processes, depending on their energies and on the medium composition (e.g. Groom et al. 2001; Nagamine 2003). The higher the muon energy, the farther the muon travels, but also the lower the muon flux. High energy muons can penetrate up to kilometres of rocks at $\mathrm{TeV}$ energies. Furthermore, due to their strong relativistic boost, they travel along straight (ballistic) paths. Muon telescopes, or muon detectors, are used to detect them and reconstruct their trajectories after the muons have crossed their target (Nagamine et al. 1995). The backward extrapolation of the muon trajectory up to the target is referred to as the line of sight. The rate of muons crossing the target along this direction depends, to first order, on the subsurface density integrated along the line of sight. Rock composition can modify this rate by up to 10-15 per cent (Lechmann et al. 2018), particularly if rocks are water saturated or not. Nagamine et al. (1995) published the first muographic measurements that were used to probe the inner structure of a volcano, from experiments on Mount Tsukuba and Mount Asama in Japan. In the last two decades, muography has been developed for the density imaging (e.g. Tanaka et al. 2001; Lesparre et al. 2012; Kusagaya \& Tanaka 2015) and monitoring (e.g. Tanaka et al. 2014; Jourde et al. 2015b) of volcanoes. Muon tomography, consisting of combining muographies from several viewpoints, allows the reconstruction the $3-\mathrm{D}$ density distribution. To conduct a muon tomography, Nagahara \& Miyamoto (2018) used the method of filtered back projection, which has the advantage of not relying on any a priori information. They show that the method requires data from at least a dozen of viewpoints to allow a proper 3-D reconstruction, which is for now impractical. Indeed, the main limitations of muon tomography are the number of available telescopes, the acquisition duration and the ability to only image densities above the horizontal plane passing through the telescope. Because of these limitations, it is helpful to combine muography with other types of data.

Both gravimetry and muography being independent and complementary methods sensitive to the subsurface densities, they have the potential to help each other through a joint inversion scheme to better constrain the 3-D density models and perform a density tomography. Both types of observations can be linearly related to densities, but the inverse problem is ill-posed, requiring additional constraints, such as a regularity of the solution relative to a prior density model. Whether the problem searched is formulated in a Bayesian framework or as a misfit minimization problem, there is a need to determine some a priori parameters tuning the regularization, further referred to as hyperparameters. So far, the determination of the hyperparameters in joint inversions of muography and gravimetric data has not been fully addressed. Nishiyama et al. (2014b) use a Bayesian approach and determine the a priori density standard deviation and correlation length by maximizing the similarity of the reconstructed densities with a given checkerboard model. Because the determined a priori values depend on the density contrast and the length of the checkerboard cells, there is no guarantee that the determined parameters apply to in-situ reconstructions. Nishiyama et al. (2017) choose the a priori density mean and standard deviation according to the muographic observations and arbitrarily fix the correlation length. Jourde et al. (2015a) and Rosas-Carbajal et al. (2017) use a non-Bayesian framework, regularizing the inversion with a prior model, weighted to counteract the physical decrease of sensitivity away from the data. Rosas-Carbajal et al. (2017) invert for a density model as well as a constant possible offset between the density inferred by muographic data and the density inferred by gravimetric data. The authors determine the weight of the smoothing by using a classical L-curve scheme in which the best compromise between the data fit and the weight of the prior model is obtained. Noteworthy, the inversion results show artefacts related to the muographic acquisition geometry: resulting densities tend to smear out along the telescopes' lines of sight. The determination of the regularization parameters should be further studied. Indeed it is recognized as a key issue in many geophysical problems with similar formulations such as the determination of slip distribution of faults (Fukuda \& Johnson 2008), or the joint determination of density and seismic slowness (Coutant et al. 2012). Comparing solutions determined using L-curves, Akaike Bayesian information criteria, cross-validation and fully Bayesian criteria, Fukuda \& Johnson (2008) have shown that there is no rational way of selecting a single value from the L-curve, and that the cross-validation and the fully Bayesian criteria provide more reliable results.

In this paper, we present a robust workflow to linearly invert muographic and gravimetric data using a Bayesian framework (Tarantola \& Valette 1982), suitable even when a limited number of muon telescopes is available. A realistic synthetic model adopting the topography of the Puy de Dôme volcano in the Chaîne des Puys in France and the acquisition geometry of gravimetric and muographic campaigns conducted on this volcano is used (Fig. 1). We use a homogeneous a priori density model and impose smoothing using a Gaussian spatial correlation function. We systematically explore the two inversion hyperparameters that tune the result of the inversion, that is, the density a priori standard deviation and the spatial correlation length, and we discuss the use of L-curves, Leave One Out (LOO) criterion and $k$-fold Cross-Validation Sum of Squares (CVSS; e.g. Wahba 1990; Augier 2011; James et al. 2013) to determine the optimal set of hyperparameters in a robust way and with limited artefacts related to the data acquisition. A realistic synthetic model adopting the topography of the Puy de Dôme volcano in the Chaîne des Puys in France and the acquisition geometry of gravimetric (Portal et al. 2016) and muographic (Ambrosino et al. 2015) campaigns conducted on this volcano is used (Fig. 1). We perform the following inversions: (i) independent inversion of gravimetric data, (ii) independent inversion of muographic data from a single viewpoint, (iii) joint inversion of gravimetric data and muographic data from a single viewpoint, (iv) independent inversion of muographic data from three viewpoints and (v) joint inversion of gravimetric data and muographic data from three viewpoints. We show the potential of the Bayesian framework for the inversion of muographic data. By comparing joint inversion results to independent gravimetric inversion results, we also assess the contribution of the muographic data to the recovery of the true density distribution. We illustrate the behaviour of the inversions for both one and three muographic viewpoints. Finally, we test the influence of an offset between the averaged density inferred from muography and the averaged density inferred from gravimetry.

\section{METHOD}

We describe the medium using a 3-D cubic mesh of equally spaced nodes of densities $\rho$. The density at any point with coordinates $(x$, $y, z)$ in the medium is obtained by trilinear interpolation of the densities of the eight surrounding nodes so that the density varies continuously in the medium. 


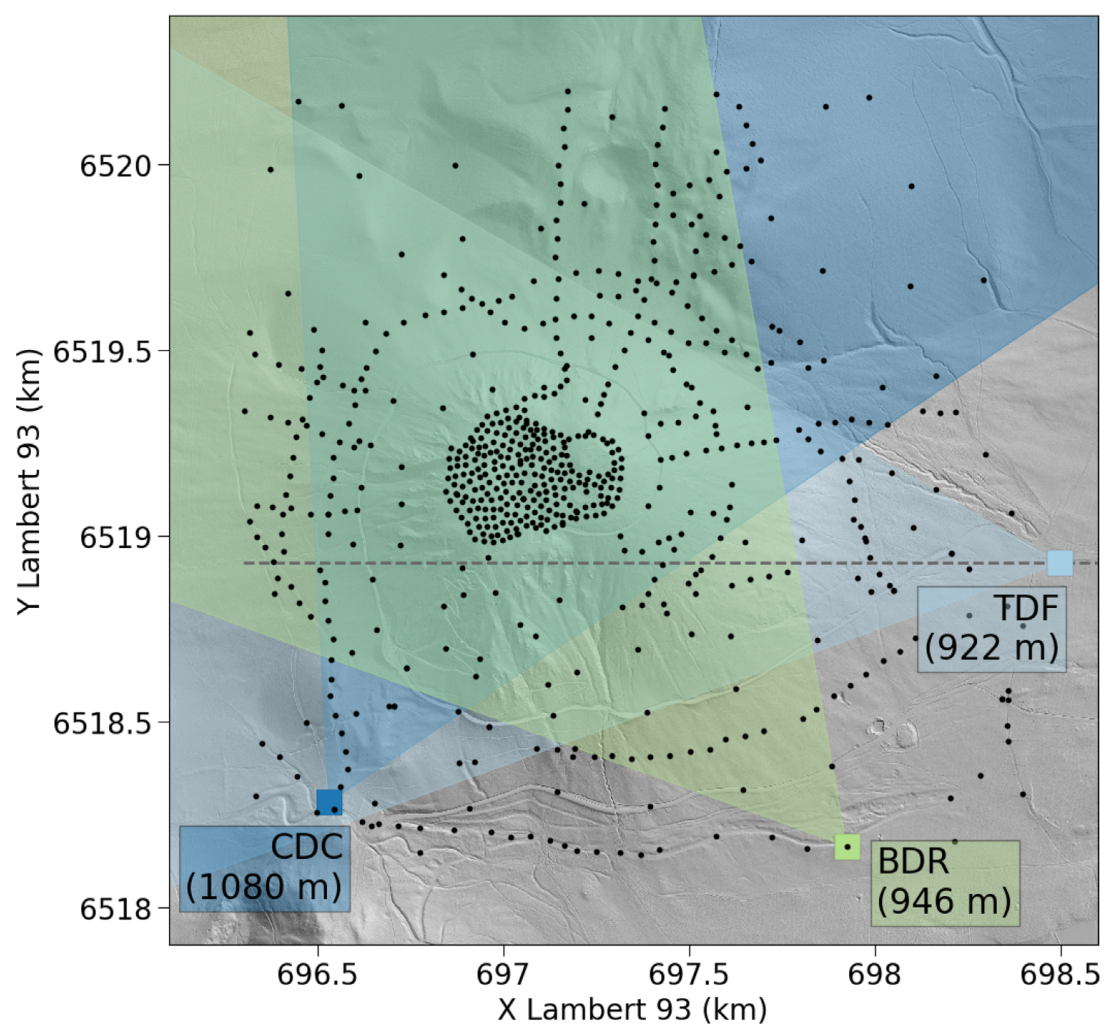

Figure 1. Shaded topography map of the Puy de Dôme volcano with the location of the gravimetric data (black dots) and of the muographic viewpoints CDC, TDF and BDR used in this study. The grey dotted line shows the location of the section used in Fig. 2.

\subsection{Forward modelling}

\subsubsection{Gravimetry}

The gravimetric anomaly $g$ produced by a volume $V$ of density $\rho(x$, $y, z)$ at a given data location $\left(x_{0}, y_{0}, z_{0}\right)$ is given by

$$
\begin{aligned}
& g\left(x_{0}, y_{0}, z_{0}\right) \\
& \quad=\mathcal{G} \iiint_{V} \frac{\rho(x, y, z)\left(z-z_{0}\right)}{{\sqrt{\left(x-x_{0}\right)^{2}+\left(y-y_{0}\right)^{2}+\left(z-z_{0}\right)^{2}}}^{3}} \mathrm{~d} x \mathrm{~d} y \mathrm{~d} z,(1)
\end{aligned}
$$

where $\mathcal{G}=6.67 \times 10^{-11} \mathrm{~m}^{3} \mathrm{~kg}^{-1} \mathrm{~s}^{-2}$ is the universal gravitational constant. The forward gravimetric computation is made using the method and the associated code of Coutant et al. (2012) described in Barnoud et al. (2016). The integration over the volume is made numerically over $x$ and $y$ and analytically over $z$. The topography is taken into account with the same resolution as the input digital terrain model in the vicinity of the gravimetric stations and is averaged with a larger step farther away.

As $\rho(x, y, z)$ can be expressed as a linear combination of densities at nodes $\rho$, the modelled gravimetric data $\boldsymbol{g}$ are also linearly related to the densities at nodes $\rho$ :

$$
\mathrm{G} \rho=g
$$

via the sensitivity matrix $\mathbf{G}$ of elements $\mathbf{G}_{i j}$ that contain the contribution of each node $j$ to each gravimetric data $i$ (Fig. 2a).

\subsubsection{Muography}

Muography provides estimates of averaged densities along lines of sight. These density estimates are retrieved from the flux of muons crossing the medium and observed in conic bins of given widths of azimuth and elevation, assuming that the estimates are uniform averages of the densities over the solid angles. To take into account the sensitivity to all the density nodes in a given cone, we integrate numerically in azimuth and elevation over the cone using a beam of lines of sight along which the density is integrated. The averaged density $\varrho$ as seen from a muon detector located in $\left(x_{0}, y_{0}, z_{0}\right)$ and in a cone with the azimuths and elevations centred on $\left(\alpha_{0}, \beta_{0}\right)$ is expressed as

$\varrho\left(x_{0}, y_{0}, z_{0}, \alpha_{0}, \beta_{0}\right)=\frac{\sum_{\alpha} \sum_{\beta} \int_{r} \rho(\alpha, \beta, r) \mathrm{d} r}{\sum_{\alpha} \sum_{\beta} \int_{r} \mathrm{~d} r}$,

where $\alpha$ and $\beta$ are the azimuths and elevations of the beam lines of sight and $r$ is the length travelled in the medium. The integration along each line of sight can be performed either numerically or analytically.

With the density in the mesh represented as a linear combination of densities at nodes, the averaged densities $\varrho$ inferred from muography can also be written as a linear combination of the densities at the nodes, similar to the gravimetric case

$\mathbf{M} \rho=\varrho$

via the sensitivity matrix $\mathbf{M}$ whose elements $\mathbf{M}_{i j}$ contain the contribution of each node of density $j$ to the averaged density in each cone $i$. The sensitivity of a muographic measurement to a node of density decreases with the distance of the node to the muon detector (Fig. 2b). $\mathbf{M}$ is a sparse matrix, in contrast to the gravimetric sensitivity matrix $\mathbf{G}$ which is full. 
(a) Sensitivity of a gravimetric measurement

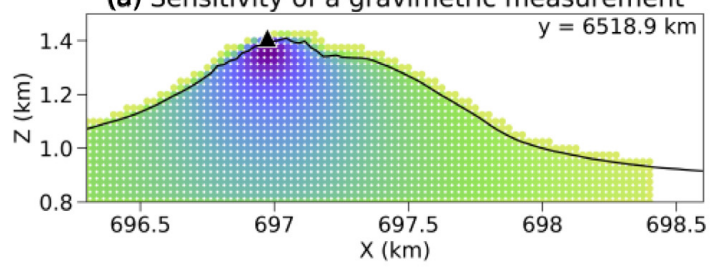

(b) Sensitivity of a muographic measurement

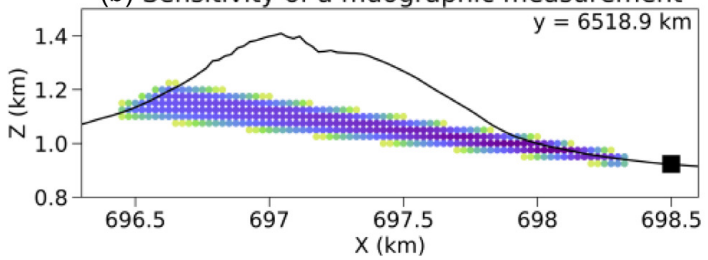

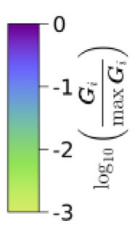

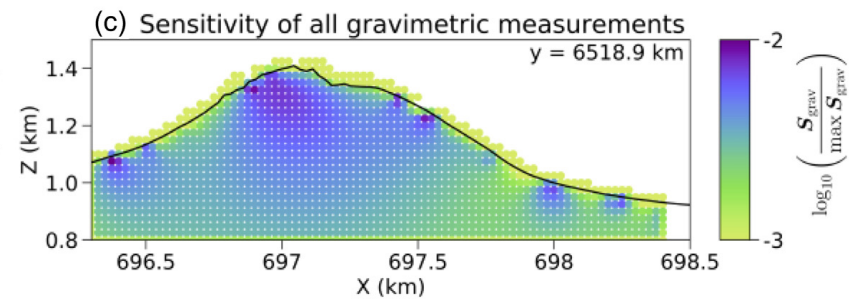

(d) Sensitivity of all muographic measurements

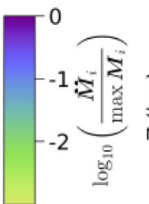

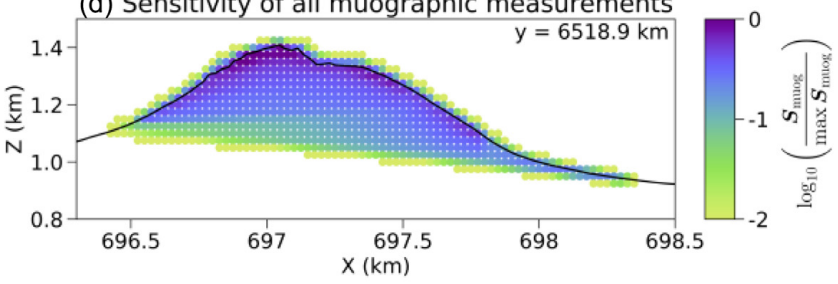

Figure 2. Sensitivities of the gravimetric and muographic data of the Puy de Dôme. The west-east cross-section is located on Fig. 1. (a) Normalized sensitivity of a single gravimetric measurement. Black triangle: gravity station location. (b) Normalized sensitivity of a single muographic measurement. Black square: location of a muographic measurement looking in a $3^{\circ} \times 3^{\circ}$ cone. (c) Normalized cumulative sensitivity of all gravimetric data to density nodes. (d) Normalized cumulative sensitivity of all muographic data to density nodes. $\boldsymbol{G}_{i}$ is the $i$ th line of the sensitivity matrix $\mathbf{G}$, corresponding to the $i$ th datum. $\boldsymbol{M}_{i}$ is the $i$ th line of the sensitivity matrix $\mathbf{M}$, corresponding to the ith datum. The sensitivities $\boldsymbol{S}_{\text {grav }}$ and $\boldsymbol{S}_{\text {muog }}$ of all data are computed by summing the lines of the sensitivity matrices, following eqs (16) and (17), respectively.

\subsection{Inversion method}

Both the gravimetric and the muographic problems being linear, with respect to the same physical property of rocks, in this case density $\rho$, the joint problem is expressed as

$\left[\begin{array}{l}\mathbf{G} \\ \mathbf{M}\end{array}\right] \rho=\left[\begin{array}{l}\mathbf{g} \\ \varrho\end{array}\right]$

Setting the vector of observed data $\boldsymbol{d}=\left[\begin{array}{ll}\boldsymbol{g}_{\text {obs }} & \boldsymbol{\varrho}_{\text {obs }}\end{array}\right]^{T}$ and the sensitivity matrix with the contribution of each node of density to each data $\mathbf{A}=[\mathbf{G} \mathbf{M}]^{T}$, the inverse problem to solve is

$\boldsymbol{d}=\mathbf{A} \rho+\epsilon$

where $\epsilon$ accounts for measurement and modelling errors.

To solve the inverse problem in eq. (6), we use a Bayesian formalism, following Tarantola (2005), which has the advantage of taking into account the errors on the data and to easily include an a priori model to regularize the inversion. We assume a zero-mean normal distribution of the data errors $\epsilon$ with a covariance matrix $\mathbf{C}_{\mathrm{D}}, \mathcal{N}\left(0, \mathbf{C}_{\mathrm{D}}\right)$, and we write the likelihood as

$p(\boldsymbol{d} \mid \boldsymbol{\rho}) \propto \exp \left(-\frac{1}{2}(\boldsymbol{d}-\mathbf{A} \boldsymbol{\rho})^{T} \mathbf{C}_{\mathrm{D}}^{-1}(\boldsymbol{d}-\mathbf{A} \boldsymbol{\rho})\right)$,

where $\mathbf{C}_{\mathrm{D}}$, the data covariance matrix, contains the data variances $\sigma_{d}^{2}$ on its diagonal and optionally covariances between data on its off-diagonal terms. This might be the case for muographic data depending on the processing applied to obtain the averaged densities. In this paper, we work with synthetic data sets and only consider independent data, leading to a diagonal data covariance matrix. We also assume an a priori normal probability density distribution for the densities at nodes, $\mathcal{N}\left(\bar{\rho}_{\text {prior }}, \mathbf{C}_{\mathrm{P}}\right)$, with an average density $\rho_{\text {prior }}$ and a covariance matrix $\mathbf{C}_{P}$

$p(\rho) \propto \exp \left(-\frac{1}{2}\left(\rho-\rho_{\text {prior }}\right)^{T} \mathbf{C}_{\mathrm{P}}^{-1}\left(\rho-\rho_{\text {prior }}\right)\right)$.

The a priori density covariance matrix $\mathbf{C}_{\mathrm{P}}$ is a full matrix that includes the standard deviation $\sigma_{\rho}$ on the a priori densities $\boldsymbol{\rho}_{\text {prior }}$ and a Gaussian spatial correlation function

$c_{\lambda}(d)=\exp \left(-\frac{d^{2}}{\lambda^{2}}\right)$

where $d$ is the distance between two nodes of the model and $\lambda$ is the spatial correlation length that controls the smoothness of the density model.

Bayes theorem states that the a posteriori probability distribution of the model densities is

$p(\rho \mid \boldsymbol{d})=\frac{p(\boldsymbol{d} \mid \rho) p(\rho)}{p(\boldsymbol{d})}$

where $p(\boldsymbol{d})$ is the marginal likelihood that does not depend on $\rho$. Hence, the a posteriori probability density function of densities is also normal and such that

$p(\boldsymbol{\rho} \mid \boldsymbol{d}) \propto \exp \left(-\frac{1}{2} \phi(\rho)\right)$

with the objective function $\phi$ given by

$$
\begin{aligned}
\phi(\rho) & =(\boldsymbol{d}-\mathbf{A} \rho)^{t} \mathbf{C}_{\mathrm{D}}^{-1}(\boldsymbol{d}-\mathbf{A} \rho)+\left(\rho-\rho_{\text {prior }}\right)^{t} \mathbf{C}_{\mathrm{P}}^{-1}\left(\rho-\rho_{\text {prior }}\right) \\
& =\|\boldsymbol{d}-\mathbf{A} \rho\|_{\mathrm{D}}^{2}+\left\|\rho-\rho_{\text {prior }}\right\|_{\mathrm{P}}^{2} .
\end{aligned}
$$

The first term represents the fit of the data. In the following, we will note $\chi^{2}=\frac{1}{n_{d}}\|\boldsymbol{d}-\mathbf{A} \boldsymbol{\rho}\|_{\mathrm{D}}^{2}$ the fit normalized by the number of data $n_{d}$, and we will refer to $\chi^{2}$ as the data misfit. The second term accounts for the proximity of the model to the prior $\rho_{\text {prior }}$, and the correlation between nearby parameters. The second term will be further referred to as the model regularization.

Maximizing the posterior probability density function is equivalent to minimizing the objective function $\phi(\rho)$ of eq. (12) and leads to a unique solution that can be written (Tarantola 2005)

$\tilde{\rho}=\rho_{\text {prior }}+\mathbf{C}_{\mathrm{P}} \mathbf{A}^{t}\left(\mathbf{A} \mathbf{C}_{\mathrm{P}} \mathbf{A}^{t}+\mathbf{C}_{\mathrm{D}}\right)^{-1}\left(\boldsymbol{d}-\mathbf{A} \rho_{\text {prior }}\right)$.

The estimated density $\tilde{\rho}$ is the centre of the a posteriori density distribution $p(\boldsymbol{\rho} \mid \boldsymbol{d})$, with the associated a posteriori density covariance matrix (Tarantola 2005)

$\widetilde{\mathbf{C}}_{\mathrm{P}}=\mathbf{C}_{\mathrm{P}}-\mathbf{C}_{\mathrm{P}} \mathbf{A}^{t}\left(\mathbf{A} \mathbf{C}_{\mathrm{P}} \mathbf{A}^{t}+\mathbf{C}_{\mathrm{D}}\right)^{-1} \mathbf{A} \mathbf{C}_{\mathrm{P}}$. 
The diagonal terms of $\widetilde{\mathbf{C}}_{\mathrm{P}}$ are the variances ${\widetilde{\sigma_{\rho}}}^{2}$ of the estimated densities at nodes $\tilde{\boldsymbol{\rho}}$.

The solution of the inversion, eqs (13) and (14), is tuned by the two hyperparameters included in the a priori covariance matrix $\mathbf{C}_{\mathrm{P}}$ : the standard deviation $\sigma_{\rho}$ of the a priori density distribution and the spatial correlation length $\lambda$. To estimate the optimal set of hyperparameters, $\left(\sigma_{\rho}, \lambda\right)$, we explore them in a systematic way and compare different criteria. On the one hand, we use classical L-curves, where the optimal set of $\left(\sigma_{\rho}, \lambda\right)$ usually corresponds to the corner of the curve representing the model regularization versus the data misfit, indicating that the best balance between the data fit and the model regularity is achieved. Typically, the larger $\lambda$ or the smaller $\sigma_{\rho}$, the smoother the solution, but the larger the data misfit. Following Harris \& Segall (1987), we also use a modified L-curve, where we assume that the optimal $\left(\sigma_{\rho}, \lambda\right)$ minimizes the a posteriori density standard deviation as well as the data misfit. On the other hand, we use $k$-fold CVSS, including the LOO case (e.g. Wahba 1990; Augier 2011; James et al. 2013). The CVSS method is based on the assumption that appropriate values of the hyperparameters lead to a resulting model that is able to accurately predict new data. The $k$-fold CVSS consists in separating the data in $k$ subsets and performing $k$ inversions excluding one of the subsets each time. The excluded subset is modelled using the inversion result. To assess how well we recover the initial synthetic model, the Mean Square Error (MSE) between modelled and observed data of this subset is evaluated. The value to minimize is then the sum or the average of the $k$ successive MSEs. Here, we use a weighted MSE that includes a normalization by the data errors, to account for the different physical quantities involved in the joint inversion, that is, densities for the muographic data and accelerations for the gravimetric data. The quantity to minimize is therefore (James et al. 2013)

$C_{k}\left(\sigma_{\rho}, \lambda\right)=\frac{1}{k} \sum_{l=1}^{k} \chi_{l}^{2}=\frac{1}{k n_{k}} \sum_{l=1}^{k}\|\boldsymbol{d}-\mathbf{A} \rho\|_{\mathrm{D}_{l}}^{2}$,

where $n_{k}$ is the total number of gravity and muography observed data in each data subset $\mathrm{D}_{l}$. We compute $C_{k}\left(\sigma_{\rho}, \lambda\right)$ for $k$ equal to a quarter of the data $(k=4)$ and to all but one data $(k=n)$, the latter case corresponding to the so-called LOO solution. The data are randomly distributed between the subsets.

\section{APPLICATION TO A REALISTIC SYNTHETIC CASE}

\subsection{Setup}

Here, we apply the inverse scheme to a realistic synthetic case in which we use the topography and acquisition geometry of recent gravimetry and muography campaigns at the Puy de Dôme volcano. The gravimetric data (Fig. 1) consist of 650 points (Portal et al. 2016). The synthetic gravimetric data correspond to a free-air anomaly, without any regional field component. Three muography viewpoints are used. Two of the muographic viewpoints (CDC: Col de Ceyssat and TDF: telecommunications room) are actual measuring stations from preliminary campaigns on the Puy de Dôme volcano (Ambrosino et al. 2015) while the third one (BDR: Bois de Rochetoux) is a fictitious viewpoint added for the purposes of this study. The muographic synthetic data consist in 2067 averaged densities in cones of $1^{\circ} \times 1^{\circ}$.

The volume is discretized using a mesh of $n_{\rho}=209525$ density nodes with a $25 \mathrm{~m}$ spacing in the three dimensions, only 107

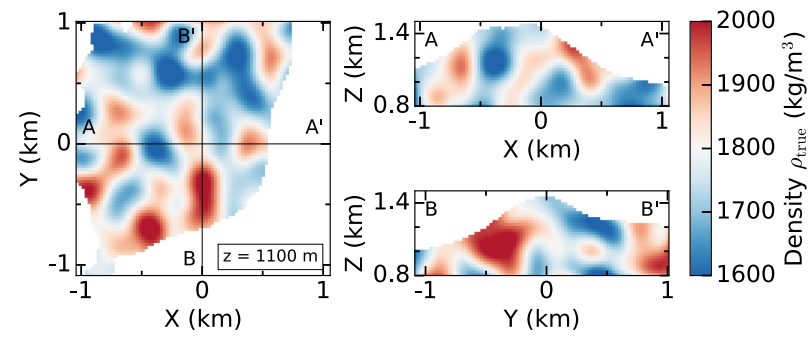

Figure 3. Synthetic density model $\rho_{\text {true }}$ used to compute the synthetic gravimetric and muographic data. The densities are constructed using a Gaussian random field with a standard deviation $\sigma_{\rho_{\text {true }}}=100 \mathrm{~kg} \mathrm{~m}^{-3}$ and a Gaussian spatial covariance with a correlation length $\lambda_{\rho_{\text {true }}}=200 \mathrm{~m}$.

164 nodes with non-zero sensitivities to be taken into account for the inversion. This implies that the covariance matrix $\mathbf{C}_{\mathrm{P}}$ used for the model regularization would account for $\sim 85$ Go of memory. The order of operations has to be handled appropriately to reduce memory needs. Based on eq. (13), the largest matrix we compute and store is the matrix product $\mathbf{C}_{\mathrm{P}} \mathbf{A}^{t}$ (Barnoud et al. 2016), only accounting for $\sim 2$ Go of memory.

The sensitivity of a gravimetric datum $i$ to the density nodes is given by the $i$ th line $\boldsymbol{G}_{i}$ of the absolute values of the sensitivity matrices $\mathbf{G}$ (Fig. 2a). Similarly, the sensitivity of a muographic datum $i$ to the density nodes is given by the $i$ th line $\boldsymbol{M}_{i}$ of the absolute values of the sensitivity matrices M (Fig. 2b). In Figs 2(c) and (d), we show the cumulated sensitivity of each node to all data. For the $n_{\text {grav }}$ gravimetric data, it is expressed as

$\boldsymbol{S}_{\text {grav }}=\sum_{i=1}^{n_{\text {grav }}} \boldsymbol{G}_{i}$,

and for the $n_{\text {muog }}$ muographic data, it is expressed as

$\boldsymbol{S}_{\text {muog }}=\sum_{i=1}^{n_{\text {muog }}} \boldsymbol{M}_{i}$.

The sensitivity of the gravimetric data is high in the top central part of the dome, thanks to a very good data coverage on the summit part of the Puy de Dôme. The sensitivity of muography increases when the depth of rock crossed by the muons decreases, hence closer to the topography. Note that the sensitivity expressed by eqs (16) and (17) corresponds to the theoretical sensitivity linked to the data distribution and does not take into account any error in the data nor prior information on densities. Therefore, it should not be seen as the expected sensitivity for the inversion results, but rather as how the data network illuminates the volume of interest.

For the purpose of this paper, we design an ideal synthetic density model with the same geometrical properties as the a priori density distribution used to regularize the inversion, that is to say a model with normally distributed densities and following a spatial Gaussian correlation function according to eqs (8) and (9). This ideal case allows us to better illustrate and discuss the methods to determine the hyperparameters.

\subsection{Construction of the synthetic model and data}

The synthetic density model (Fig. 3) is a Gaussian random model such that the densities are drawn from $\mathcal{N}\left(\bar{\rho}_{\text {true }}, \mathbf{C}_{\rho_{\text {true }}}\right)$, that is to say a normal distribution with an averaged density $\bar{\rho}_{\text {true }}=1800 \mathrm{~kg} \mathrm{~m}^{-3}$ corresponding to the estimated averaged density of the Puy de Dôme (Portal et al. 2016), a density standard deviation $\sigma_{\rho_{\text {true }}}=100 \mathrm{~kg} \mathrm{~m}^{-3}$ and following a Gaussian spatial correlation with a correlation 

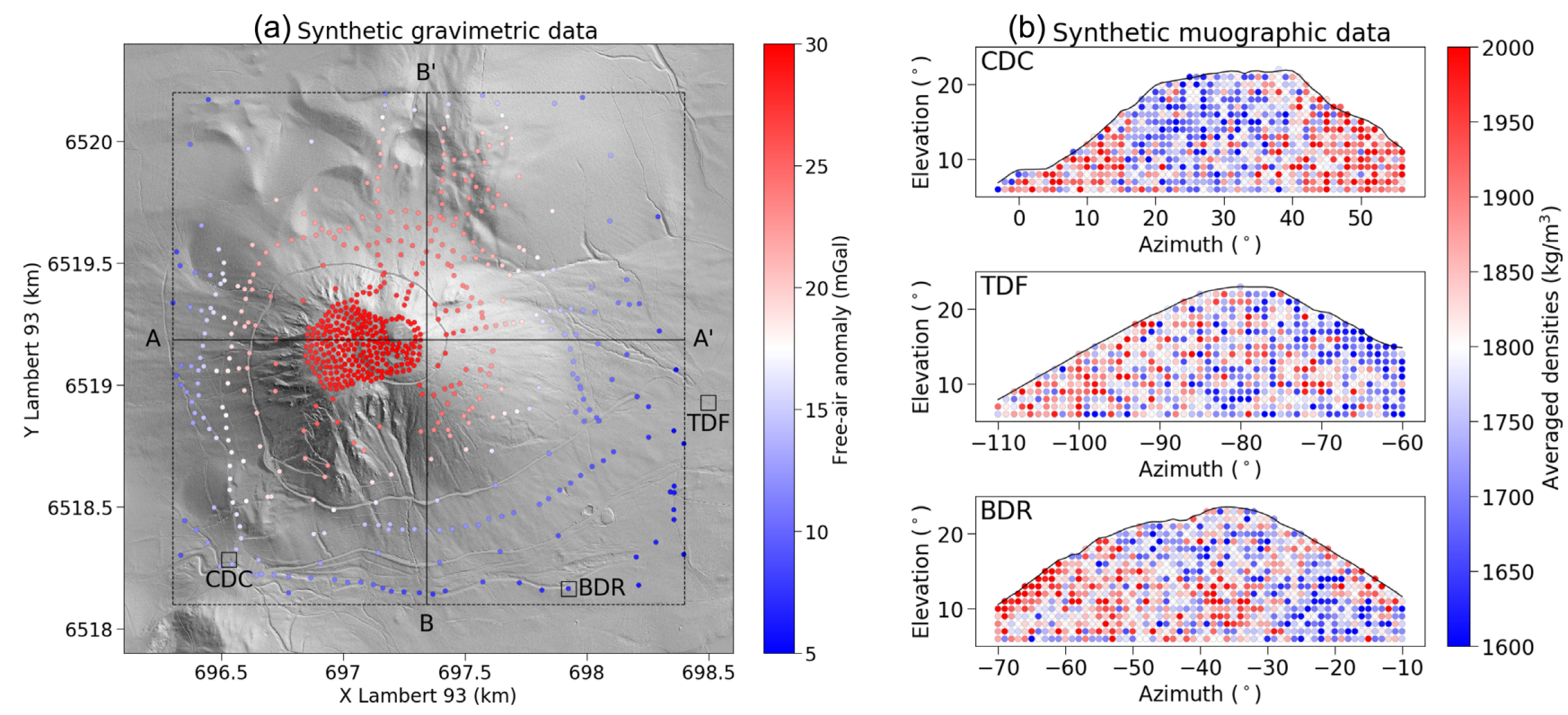

Figure 4. Synthetic data computed from the synthetic density model shown in Fig. 3. (a) Gravimetric data on the shaded topographic map. A Gaussian noise with a standard deviation of $0.1 \mathrm{mGal}$ has been added to the gravimetric data. Black squares: location of the muon detectors. The dotted square shows the limits of the inversion volume. The solid black lines show the locations of cross-sections AA' and BB' shown in this paper. (b) Muographic data from CDC, TDF and BDR viewpoints. A Gaussian noise with a standard deviation of $100 \mathrm{~kg} \mathrm{~m}^{-3}$ has been added to the data.

length $\lambda_{\text {true }}=200 \mathrm{~m}$, as indicated in the covariance matrix $\mathbf{C}_{\rho_{\text {true }}}$. In the Appendix, we recall that a synthetic density model with the required statistical properties can be constructed using

$\rho_{\text {true }}=\bar{\rho}_{\text {true }}+\mathbf{L} X$,

where $X \sim \mathcal{N}(0, \mathbf{I})$ is a random vector drawn from a zero-mean normal distribution with an identity covariance matrix and $\mathbf{L}$ is the Cholesky decomposition of the covariance matrix $\mathbf{C}_{\rho_{\text {true }}}=\mathbf{L} \mathbf{L}^{T}$ with a Gaussian correlation function of standard deviation $\sigma_{\rho_{\text {true }}}$ and of correlation length $\lambda_{\text {true }}$. For computational reasons, the random realization is generated on an subsampled mesh with a node spacing of $100 \mathrm{~m}$ and a spline interpolation is used to obtain the model over the $25 \mathrm{~m}$ mesh.

We generate synthetic data to which we add Gaussian noise with realistic standard deviations (Fig. 4). For the gravimetric data, we use a standard deviation of $0.1 \mathrm{mGal}$, which has a comparable order of magnitude as the errors from the gravimetric measurements (Portal et al. 2016). For the muographic data, we use a standard deviation of $100 \mathrm{~kg} \mathrm{~m}^{-3}$, corresponding to the order of magnitude of errors estimated for acquisition campaigns on the Puy de Dôme with an exposure of about $50 \mathrm{~d} \mathrm{~m}^{2}$ (Cârloganu et al. 2016; Cârloganu \& the TOMUVOL collaboration 2018; Niess et al. 2018b) and determined for similar campaigns on other volcanoes as well (e.g. Lesparre et al. 2012; Nishiyama et al. 2014a, 2016; Oláh et al. 2018). We assume that the data errors are accurately estimated when dealing with real data and use these values as data standard deviations in the data covariance matrix $\mathbf{C}_{\mathrm{D}}$.

\subsection{Inversion of the synthetic data}

\subsubsection{Determination of the inversion hyperparameters}

We perform the independent and joint inversions for 128 sets of hyperparameters $\left(\sigma_{\rho}, \lambda\right)$, with an a priori density standard deviation $\sigma_{\rho}$ ranging from 5 to $400 \mathrm{~kg} \mathrm{~m}^{-3}$ and a spatial correlation length $\lambda$ ranging from 50 to $800 \mathrm{~m}$. In order to identify the most suitable criterion, the L-curve and CVSS criteria are compared for the independent and joint inversions of the gravimetric and the muographic data from the three viewpoints. Comparison of the density model from the joint inversion with the true density model (Fig. 5) shows that the closest model in terms of root mean square error (RMSE) and mean absolute error (MAE) is obtained for hyperparameters $\left(\sigma_{\rho}, \lambda\right)=\left(100 \mathrm{~kg} \mathrm{~m}^{-3}, 200 \mathrm{~m}\right)$, corresponding to the parameters used to construct the synthetic density model (Fig. 3). We use this set of hyperparameters as reference in the remainder of this paper, indicated with a black dot in Figs 5-7. Note that the RMSE and MAE cannot be used as criteria to determine the hyperparameters for real data inversion as the true densities are not known.

L-curves of the model regularization versus the data misfit lead to $\left(\sigma_{\rho}, \lambda\right)=\left(400 \mathrm{~kg} \mathrm{~m}^{-3}, 50 \mathrm{~m}\right)$ for three configurations of inversions, that is, the largest tested $\sigma_{\rho}$ and the lowest tested $\lambda$ (Fig. 6, top). The associated density models appear underconstrained: they are not smooth enough leading to density anomalies spread out along the muographic lines of sight and small areas of high density intensity anomalies located right beneath the gravimetric data. As the L-curves of the model regularization versus the data misfit do not show typical L-shapes and are not conclusive to select the hyperparameters, we also plot L-curves of the a posteriori density standard deviation $\widetilde{\sigma_{\rho}}$ (averaging the variances at all nodes) versus the data misfit (Fig. 6, bottom), similarly to the L-curves used by Harris \& Segall (1987) for instance. This representation leads to $\left(\sigma_{\rho}, \lambda\right)=\left(50 \mathrm{~kg} \mathrm{~m}^{-3}, 150 \mathrm{~m}\right)$ for the gravimetric inversion, $\left(150 \mathrm{~kg} \mathrm{~m}^{-3}, 50 \mathrm{~m}\right)$ for the muographic inversion and $\left(100 \mathrm{~kg} \mathrm{~m}^{-3}, 100 \mathrm{~m}\right)$ for the joint inversion. In this case, the corresponding density model is much smoother for the gravimetric inversion, but not for the muographic inversion which is still badly constrained, with anomalies spread out along the muography lines of sight. For the joint inversion, the resulting model is better constrained than with the previous L-curve, but not as smooth as the true density model (Fig. 3). 
(a) RMSE

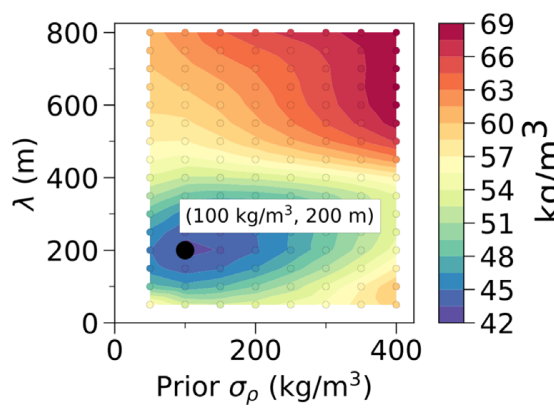

(b) MAE

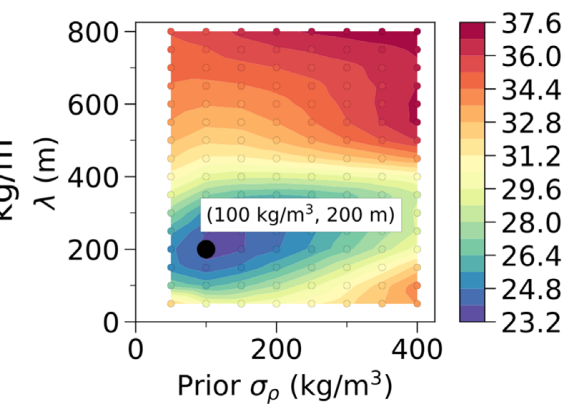

(c) Density model

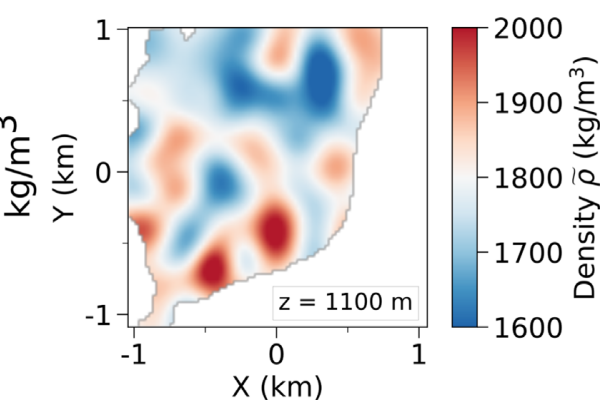

Figure 5. Systematic exploration of the inversion hyperparameters $\sigma_{\rho}$ and $\lambda$ and comparison with the true density model (Fig. 3). (a) Root-mean-square error (RMSE) between the inverted models and the true model. (b) Mean absolute error (MAE) between the inverted models and the true model. The black dots indicate the minimal RMSE and MAE, obtained for $\left(\sigma_{\rho}, \lambda\right)=\left(100 \mathrm{~kg} \mathrm{~m}^{-3}, 200 \mathrm{~m}\right)$. These values correspond to the true density model (Fig. 3$)$. We use these hyperparameter values and their associated model as reference in the following of this paper. (c) Optimal density model associated with these hyperparameter values.

Fig. 7 shows maps of the LOO criterion $C_{n}\left(\sigma_{\rho}, \lambda\right)$ and of the fourfold CVSS criterion $C_{4}\left(\sigma_{\rho}, \lambda\right)$ computed by eq. (15). The optimal density models are obtained for the hyperparameters $\sigma_{\rho}$ and $\lambda$ that minimize the functions $C_{n}\left(\sigma_{\rho}, \lambda\right)$ and $C_{4}\left(\sigma_{\rho}, \lambda\right)$ of eq. (15). Both the LOO and the fourfold CVSS criteria give hyperparameters $\left(\sigma_{\rho}, \lambda\right)$ (white dots) that slightly overestimate the reference value of $\left(100 \mathrm{~kg} \mathrm{~m}^{-3}, 200 \mathrm{~m}\right)$ (black dots) by at most $100 \mathrm{~kg} \mathrm{~m}^{-3}$ and $50 \mathrm{~m}$. The fourfold CVSS leads to values closer to the reference values than the LOO. In the three configurations of inversion, the LOO and CVSS maps have areas of minimum $C_{n}$ and $C_{4}$ that are elongated along the $\sigma_{\rho}$ axis, indicating that the spatial correlation length $\lambda$ is better constrained than the a priori density standard deviation $\sigma_{\rho}$ (Fig. 7). However, we observe that the density models associated with low LOO or CVSS criteria (areas in violet on hyperparameters maps of Fig. 7) are very similar to each other. Indeed, even when the LOO and fourfold CVSS criteria do not give the exact same hyperparameter values, the corresponding models are indistinguishable (Fig. 7) and acceptably recover the amplitudes and shapes of the synthetic density model (Fig. 3). This observation is supported by the RMSE and MAE maps that have similar shapes as the LOO and CVSS maps (Fig. 5), suggesting that the corresponding resulting models are expected to be similar to each other (Fig. 5). Besides, we no longer observe artefacts linked to the data acquisition geometry in the resulting density models, compared to the models obtained using the L-curves (Fig. 6). The LOO and CVSS criteria are therefore robust criteria and helpful tools to determine optimal values for hyperparameters.

\subsubsection{Inversion results}

In order to allow the comparison between the joint and independent inversions, we retain the results obtained using the reference hyperparameter values $\left(\sigma_{\rho}, \lambda\right)=\left(100 \mathrm{~kg} \mathrm{~m}^{-3}, 200 \mathrm{~m}\right)$, keeping in mind that density models obtained with hyperparameters inferred from LOO or CVSS criteria are very close. We show the inversion results for the five following configurations of inversion (Figs 8 and 9): the independent inversion of the gravimetric data (Figs $8 b$ and $9 b$ ); the independent inversion of the muographic data from the CDC viewpoint (Figs 8c and 9c); the joint inversion of the gravimetric data and the muographic data from the CDC viewpoint (Figs $8 \mathrm{~d}$ and 9d); the independent inversion of the muographic data from CDC, TDF and BDR viewpoints (Figs 8e and 9e); and the joint inversion of the gravimetric data and the muographic data from the three viewpoints (Figs 8f and 9f), see Fig. 1 for the locations. Horizontal and vertical cross-sections extracted from the 3 -D resulting models are shown in terms of central density $\widetilde{\rho}$ and standard deviation $\widetilde{\sigma_{\rho}}$ of the a posteriori density distribution (Figs 8 and 9, top and middle, respectively), as well as a random realization of densities drawn within this a posteriori distribution (Figs 8 and 9, bottom). The density models randomly drawn from the a posteriori distribution allow for a better visualization of the resolution of the model because well resolved areas show some spatial correlation while unresolved areas display randomly distributed densities with no spatial correlation. To quantify the differences between the results of the five inversion configurations, we compute several estimators: the RMSE and the MAE between the inverted models and the synthetic density model, the mean of the a posteriori density standard deviation at all nodes, and the data misfits (Table 1).

The independent inversion of the gravimetric data alone allows retrieving lateral density variations with the lowest a posteriori standard deviation in the upper $200 \mathrm{~m}$ below the topographic surface (Fig. 9b). The standard deviation on the a posteriori density (Fig. 9b, middle) as well as the model constructed by randomly sampling the a posteriori density distribution according to their standard deviation (Fig. 9b, bottom) show that the gravimetric inversion suitably reproduces the anomalies at shallow depth but has difficulties retrieving the shapes of the structures at depth.

The independent inversion of the muographic data from the CDC viewpoint alone spreads out the density variations along the lines of sight along which the muography is blind (Fig. 8c). The a posteriori density standard deviation shows that the densities far away from the detector are not constrained (Fig. 8c, middle). The retrieved density model is quantitatively less good than the one obtained with the independent gravimetric inversion, the RMSE and MAE with respect to the synthetic density model increasing by 49.5 and 52.8 per cent respectively (Table 1).

The result of the joint inversion of the gravimetric and the muographic data from CDC viewpoint alone (Figs 8d and 9d) shows the complementarity of the two types of data. The resolution at depth is improved and the limit of low a posteriori standard deviation at depth is extended from $\sim 200 \mathrm{~m}$ to $\sim 300 \mathrm{~m}$ below the summit. In this case, muography brings resolution at depth in the central part of the dome and helps to better localise the anomalies in the core of the dome (compare Fig. 9d middle with Figs 9b middle and 9c middle), while gravimetry constrains the parts of the model away from the muon detector and the lateral variations (Fig. 8d). Quantitatively 
(a) Gravimetric inversion

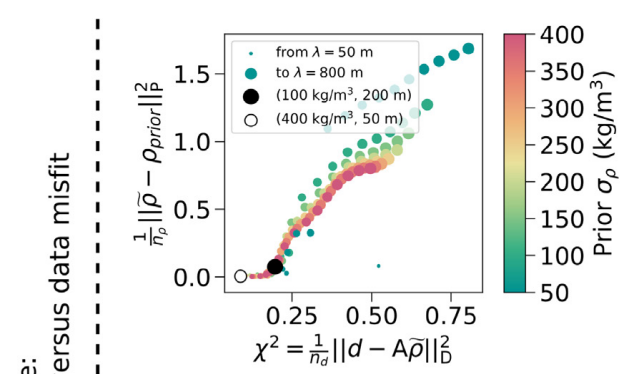

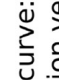

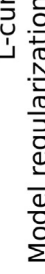
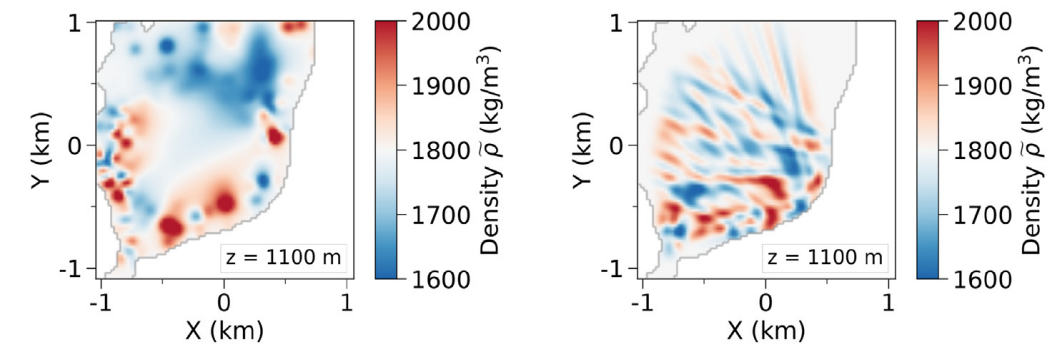

(b) Muographic inversion CDC+TDF+BDR
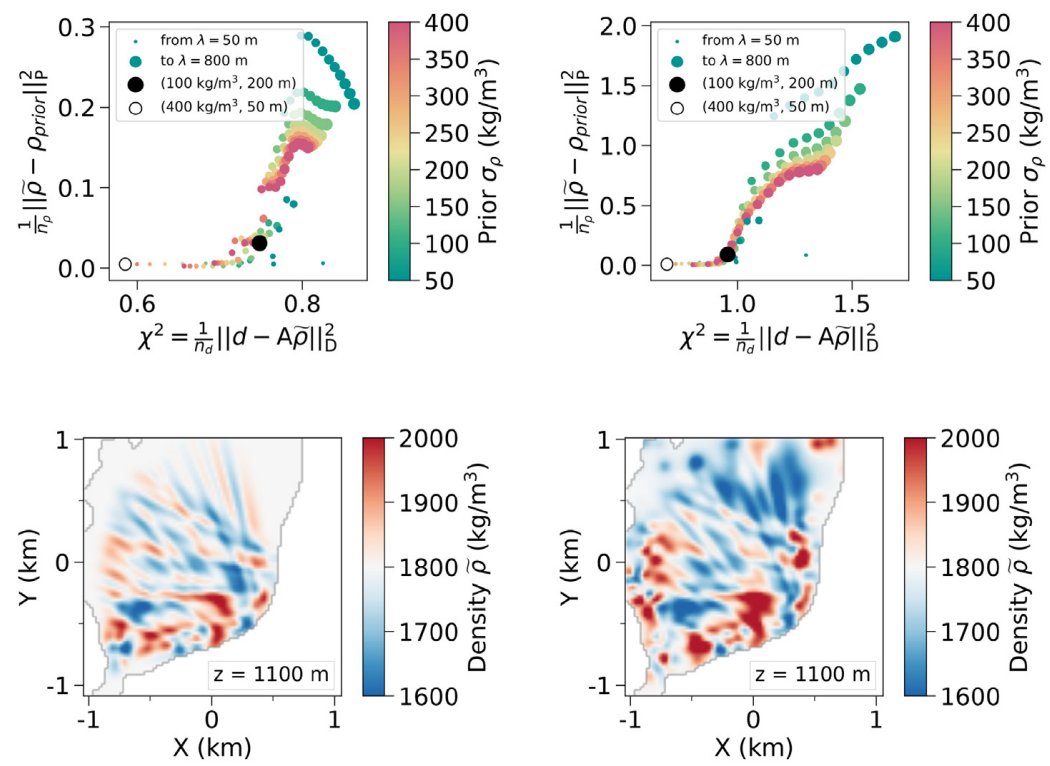
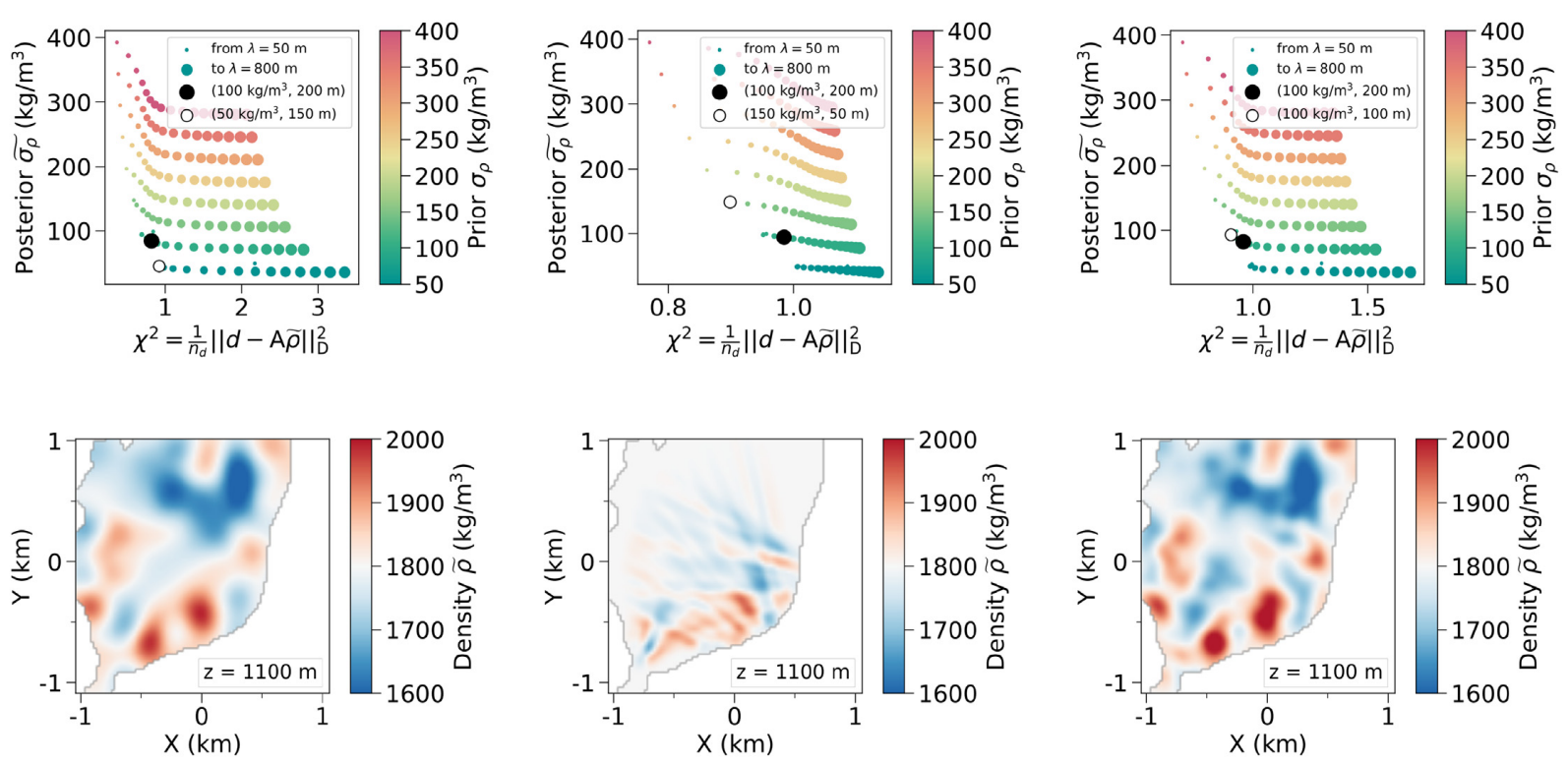

Figure 6. L-curve criteria to select the inversion hyperparameters $\sigma_{\rho}$ and $\lambda$, along with the associated optimal models (horizontal cross-sections extracted at $1100 \mathrm{~m}$ of altitude). The dot colour indicates the prior value $\sigma_{\rho}$, while the dot size is proportional to the correlation length $\lambda$. The white dots indicate the optimal values obtained for the L-curves. The corresponding $\sigma_{\rho}$ and $\lambda$ values are indicated in the legend. The black dots indicate the reference model for $\left(\sigma_{\rho}, \lambda\right)=\left(100 \mathrm{~kg} \mathrm{~m}^{-3}, 200 \mathrm{~m}\right)$ (Figs 3 and 5). Top: model regularization versus data misfit L-curve of the objective function and its associated optimal model. Bottom: posterior density distribution versus data misfit L-curve and associated optimal model. (a) Independent inversion of the gravimetric data. (b) Independent inversion of the muographic data from CDC, BDR and TDF viewpoints. (c) Joint inversion of the gravimetric and the muographic data from CDC, BDR and TDF viewpoints.

speaking, the model RMSE and MAE and the a posteriori standard deviation on density are improved with respect to the independent inversions, the addition of the muographic data improving RMSE and MAE by 2.8 and 3.1 per cent compared to the independent gravimetric inversion (Table 1).

The independent inversion of the muographic data from the three viewpoints CDC, TDF and BDR adequately retrieves the amplitudes and locations of the density anomalies in the upper part of the dome above $\sim 1000 \mathrm{~m}$ of altitude, that is, in the upper $\sim 400 \mathrm{~m}$ below the summit (Fig. 9e). This corresponds to the part of the dome illuminated by the muographic data (Fig. 2d). This result is shown as well by the a posteriori standard deviation on density (Fig. 9e, middle). Quantitatively, the RMSE and the MAE are increased by 37.2 per cent and 37.7 per cent respectively with respect to the gravimetric independent inversion (Table 1).

The joint inversion of the complete gravimetric and muographic data sets (Fig. 9f) provides the best reconstruction with RMSE and MAE improved by 7.7 per cent and 9.2 per cent respectively with respect to the gravimetric independent inversion (Table 1). Similarly, the mean a posteriori standard deviation on density is lower than 
(a) Gravimetric inversion

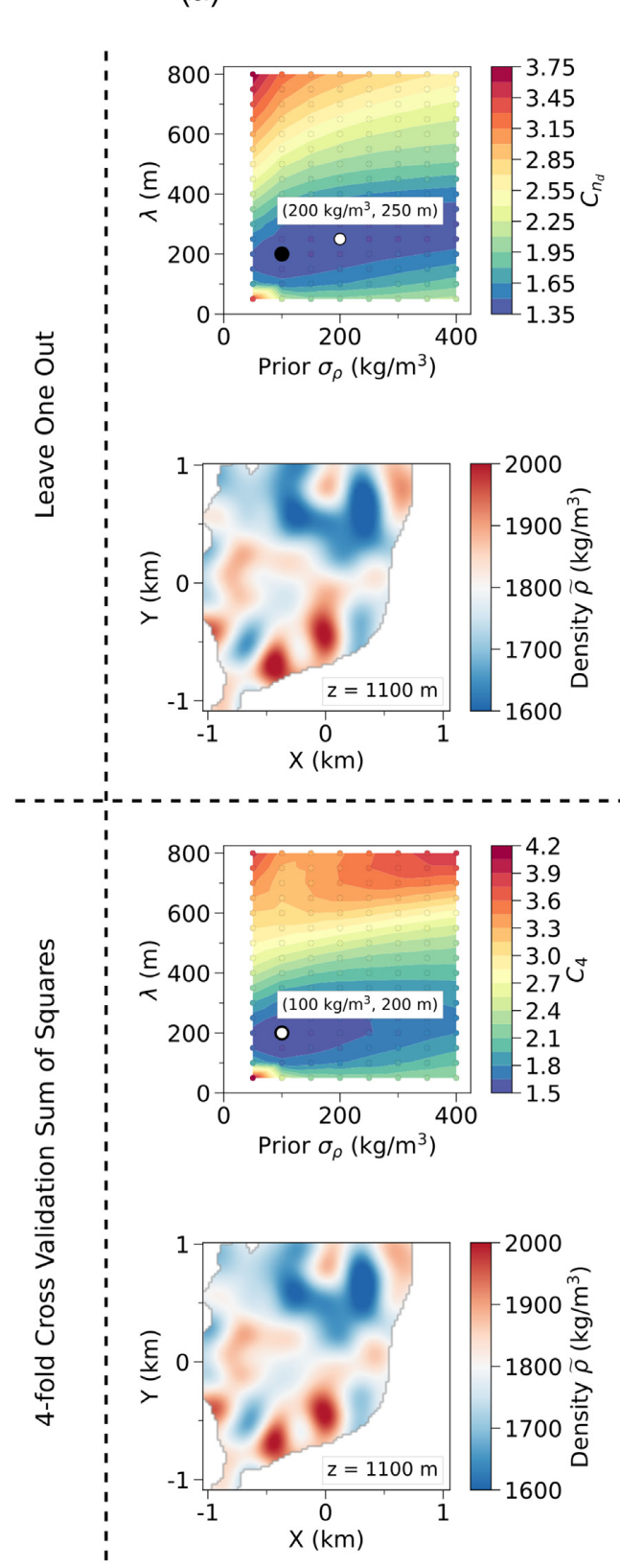

(b) Muographic inversion CDC+TDF+BDR
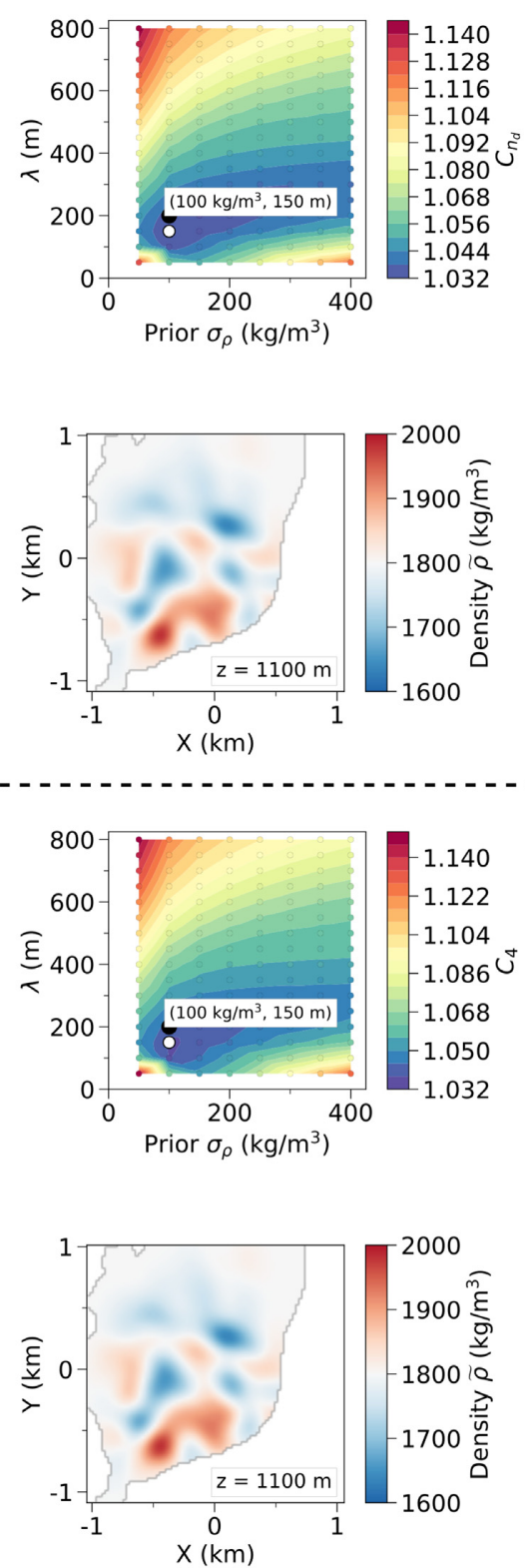

(c) Joint inversion $\mathrm{CDC}+\mathrm{TDF}+\mathrm{BDR}$
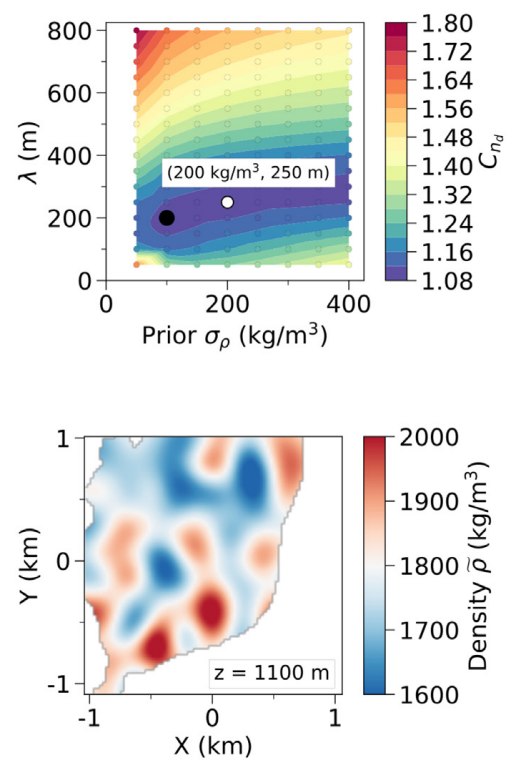

Figure 7. Leave One Out (LOO) and fourfold Cross-Validation Sum of Squares (CVSS) criteria to select inversion hyperparameters $\sigma_{\rho}$ and $\lambda$ and associated optimal models (horizontal cross-sections extracted at an altitude of $1100 \mathrm{~m}$ ). The white dots indicate the optimal values obtained from the LOO and CVSS maps. The black dots indicate the reference values $\left(\sigma_{\rho}, \lambda\right)=\left(100 \mathrm{~kg} \mathrm{~m}^{-3}, 200 \mathrm{~m}\right)$ (Figs 3 and 5). Top: LOO criterion map and its associated optimal model. Bottom: fourfold CVSS criterion map and associated optimal model. (a) Independent inversion of the gravimetric data. (b) Independent inversion of the muographic data from CDC, BDR and TDF viewpoints. (c) Joint inversion of the gravimetric and the muographic data from CDC, BDR and TDF viewpoints.

for the independent inversions (Table 1), indicating the retrieved model is better constrained. As indicated by the $\chi^{2}$ values, the data are equally well fit by both the joint and independent inversions (Table 1).

\subsection{Influence of density shifts between gravimetric data, muographic data and a priori model}

The inversion method takes into account the statistical errors on the data and on the a priori density, but it does not take into account possible systematic errors, that is, biases. Up to now, we have therefore assumed that the a priori density corresponds to the true averaged density and that gravimetric and muographic data are not biased. However, the a priori density $\bar{\rho}_{\text {prior }}$, the average density inferred from gravimetric data $\bar{\rho}_{\text {grav }}$ and the averaged density seen by muographic data $\bar{\rho}_{\text {muog }}$ might differ. Indeed, gravimetry and muography are not sensitive to the densities in the same way and data coverage illuminate the volume differently (Figs $1 \mathrm{~b}$ and c). Real gravimetric data are sensitive to the bulk rock densities of the whole Earth and are likely to be affected by the regional field that cannot be perfectly corrected, leading to a possible inaccurate determination of the averaged density, either underestimated or overestimated depending 
(a) True density model

(b) Gravimetric inversion

(c) Muographic inversion CDC

(d) Joint inversion CDC

(e) Muographic inversion
CDC+TDF+BDR

(f) Joint inversion
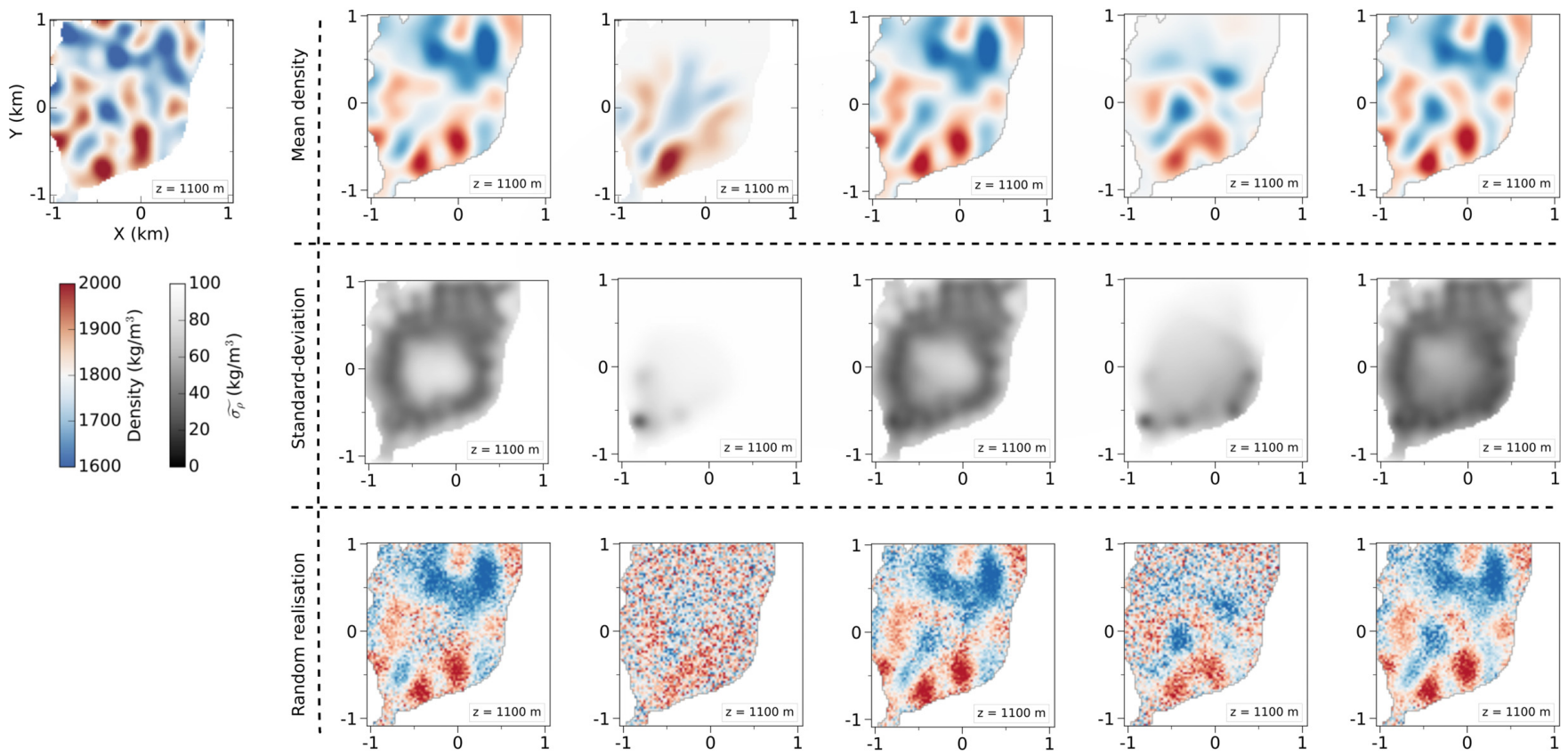

Figure 8. Horizontal cross-sections at $1100 \mathrm{~m}$ depth extracted from the 3 -D density models resulting from inversions using $\left(\sigma_{\rho}, \lambda\right)=\left(100 \mathrm{~kg} \mathrm{~m}{ }^{-3}, 200 \mathrm{~m}\right)$. (a) True density model. (b-f) A posteriori density distribution retrieved by the inversions. Top: mean density $\widetilde{\rho}$. Middle: standard deviation $\sigma_{\widetilde{\rho}}$ of the $a$ posteriori density distribution. Bottom: an example of random realization of a density model from a normal distribution centred on $\widetilde{\rho}$ and with a standard deviation of $\sigma_{\widetilde{\rho}}$. (b) Independent inversion of the gravimetric data. (c) Independent inversion of the muographic data from the CDC viewpoint only. (d) Joint inversion of the gravimetric data and the muographic data from CDC. (e) Independent inversion of the muographic data from CDC, BDR and TDF viewpoints. (f) Joint inversion of the gravimetric and the muographic data from CDC, TDF and BDR viewpoints. See Fig. 4 for the locations of the muographic viewpoints.

(a) True density model
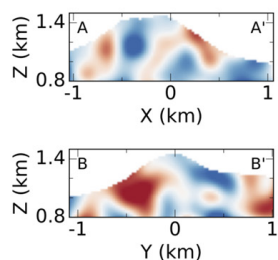

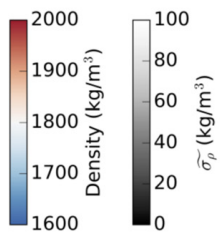

(b) Gravimetric inversion

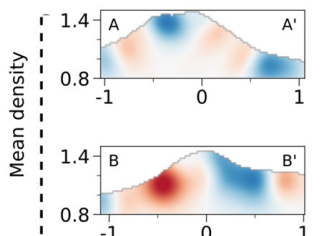

(c) Muographic inversion
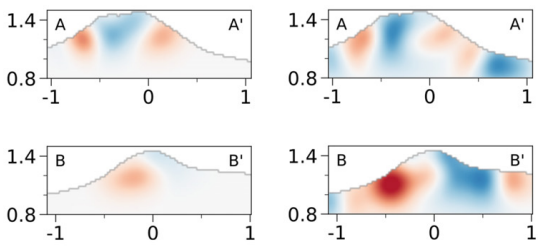

(e) Muographic inversion

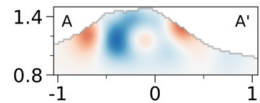

(f) Joint inversion
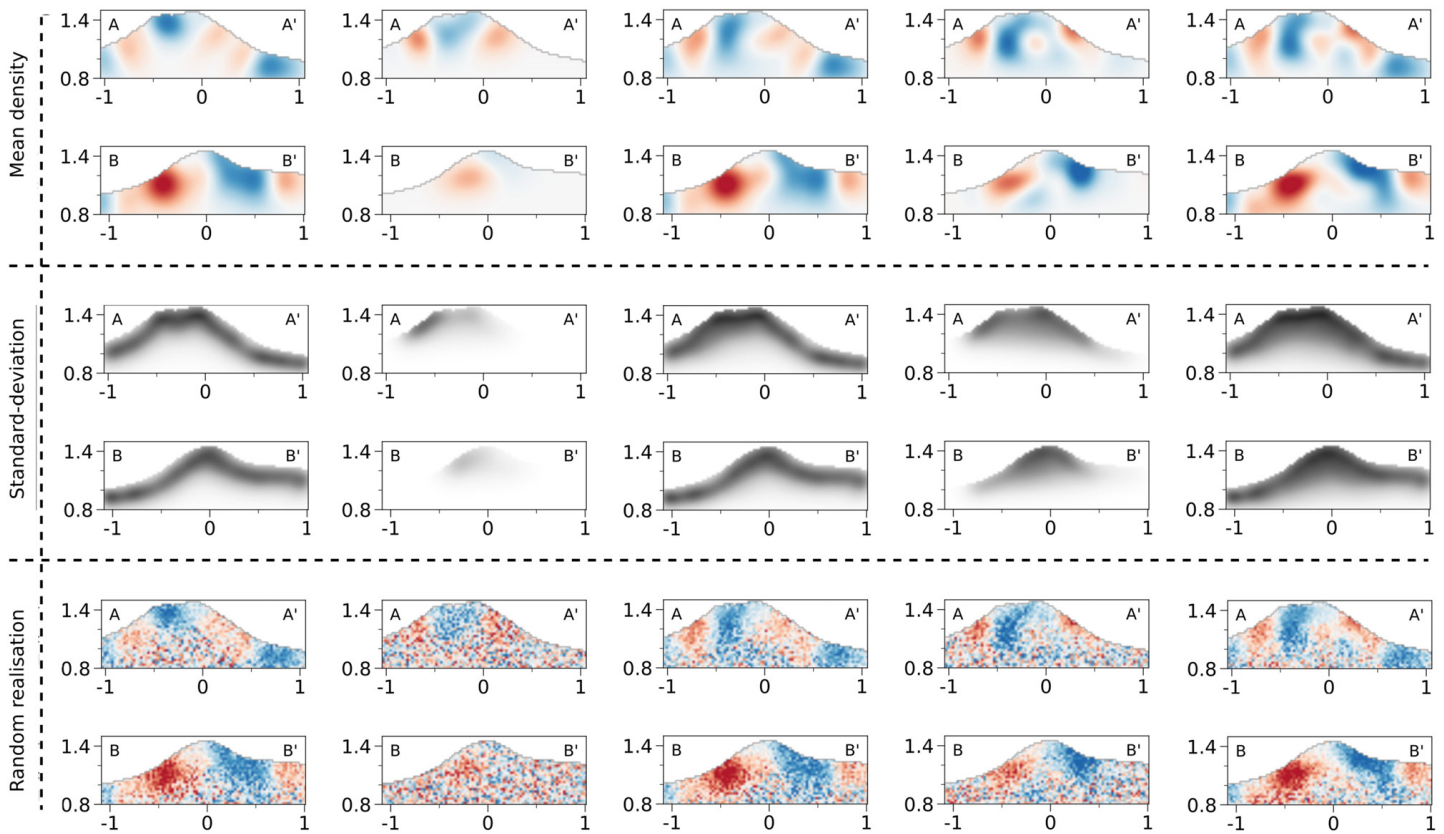

Figure 9. West-east and south-north vertical cross-sections extracted from the 3-D density models resulting from inversions using $\left(\sigma_{\rho}, \lambda\right)=$ $\left(100 \mathrm{~kg} \mathrm{~m}^{-3}, 200 \mathrm{~m}\right)$. Sections AA' and BB' are located in Fig. 3. (a) True density model. (b-f) A posteriori density distribution retrieved by the inversions. Top: mean density $\widetilde{\rho}$. Middle: standard deviation $\sigma_{\widetilde{\rho}}$ of the a posteriori density distribution. Bottom: an example of random realization of a density model from a normal distribution centred on $\widetilde{\rho}$ and with a standard deviation of $\sigma_{\tilde{\rho}}$. (b) Independent inversion of the gravimetric data. (c) Independent inversion of the muographic data from CDC viewpoint only. (d) Joint inversion of the gravimetric data and the muographic data from CDC. (e) Independent inversion of the muographic data from CDC, BDR and TDF viewpoints. (f) Joint inversion of the gravimetric and the muographic data from CDC, TDF and BDR. See Fig. 4 for locations of the sections and of the muographic viewpoints. 
Table 1. Comparison of the independent and joint inversion results (Figs 8 and 9) in terms of root-mean-square error (RMSE) and mean absolute error (MAE) with respect to the true synthetic model (Fig. 3), mean $\widetilde{\sigma_{\rho}}$ of the standard deviation on the a posteriori density model and data misfits $\chi^{2}, \chi_{\text {grav }}^{2}$ and $\chi_{\text {muog. }}^{2}$. The muographic and joint inversions use either a single muographic viewpoint CDC or the three muographic viewpoints CDC, TDF and BDR. The percentages indicate the gain with respect to the independent gravimetric inversion. A negative percentage indicates an improvement in the density model recovery, compared to the gravimetric inversion.

\begin{tabular}{|c|c|c|c|c|c|}
\hline & Gravimetric inversion & $\begin{array}{l}\text { Muographic inversion } \\
\text { CDC }\end{array}$ & $\begin{array}{c}\text { Joint inversion } \\
\text { CDC }\end{array}$ & $\begin{array}{c}\text { Muographic inversion } \\
\text { CDC+TDF+BDR }\end{array}$ & $\begin{array}{c}\text { Joint inversion } \\
\text { CDC+TDF+BDR }\end{array}$ \\
\hline $\operatorname{RMSE}\left(\mathrm{kg} \mathrm{m}^{-3}\right)$ & 65.3 & 97.6 (49.5 per cent) & $63.5(-2.8$ per cent $)$ & 89.6 (37.2 per cent) & $60.3(-7.7$ per cent $)$ \\
\hline $\operatorname{MAE}\left(\mathrm{kg} \mathrm{m}^{-3}\right)$ & 50.9 & 77.8 (52.8 per cent $)$ & $49.3(-3.1$ per cent $)$ & 70.1 (37.7 per cent) & $46.2(-9.2$ per cent $)$ \\
\hline$\chi^{2}=\frac{1}{n_{d}}\|\boldsymbol{d}-\mathbf{A} \boldsymbol{\rho}\|_{\mathrm{D}}^{2}$ & 0.905 & 0.958 & 0.939 & 0.992 & 0.978 \\
\hline$\chi_{\mathrm{grav}}^{2}=\frac{1}{n_{\mathrm{grav}}}\|\boldsymbol{g}-\mathbf{G} \boldsymbol{\rho}\|_{\mathrm{D}}^{2}$ & 0.905 & & 0.910 & & 0.911 \\
\hline
\end{tabular}

on the real density distribution in the real Earth and on the data coverage. Muography is sensitive to second order to the medium composition, such as the water content (e.g. Lechmann et al. 2018). Real muographic data might be contaminated by non-ballistic muons (Nishiyama et al. 2014a, 2016), arising from the interactions of muons with matter. This contamination strongly depends on the shape of the target (Niess et al. 2018b). These non-ballistic muons generally lead to underestimated densities (Nishiyama et al. 2016; Rosas-Carbajal et al. 2017).

Here, we perform the joint inversions, with one and three muographic viewpoints, shifting alternately the a priori density $\bar{\rho}_{\text {prior }}$, the density inferred from the gravimetric data $\bar{\rho}_{\text {grav }}$ and the averaged density inferred from the muographic data $\bar{\rho}_{\text {muog }}$ by a plausible amount of $200 \mathrm{~kg} \mathrm{~m}^{-3}$ with respect to the true averaged density $\bar{\rho}_{\text {true }}=1800 \mathrm{~kg} \mathrm{~m}^{-3}$. Note that these tests would remain valid whatever the initial value of $\bar{\rho}_{\text {true }}$. Similarly as for the unbiased data sets, the CVSS criterion maps show that the spatial correlation length $\lambda$ is better constrained than the a priori density standard deviation $\sigma_{\rho}$ (Fig. 10). Even though the retrieved optimal hyperparameter values are shifted from the reference values, they still lie within the area of lowest values so that the resulting density models (Fig. 10) are nearly identical to the ones obtained using the reference hyperparameter values. Interestingly, the hyperparameters are not better constrained when using three muographic viewpoints (Fig. 10, right) than when using a single muographic viewpoint (Fig. 10, left). For each inversion configuration, we show the density model resulting from the optimal hyperparameters derived from the fourfold CVSS criterion (Fig. 11).

First, we illustrate the behaviour of the inversion when the $a$ priori density $\bar{\rho}_{\text {prior }}$ overestimates or underestimate the true averaged density $\bar{\rho}_{\text {true }}$ by $200 \mathrm{~kg} \mathrm{~m}^{-3}$ (Figs $11 \mathrm{a}$ and b). Comparison with the results of the unbiased inversions (Fig. 9) shows that the location, the geometry and the amplitudes of the density anomalies are equally well reproduced in the upper part of the model which is well resolved by the data, with slightly lower amplitudes.

Then, we show the behaviour of the inversion when the averaged densities $\bar{\rho}_{\text {grav }}$ and $\bar{\rho}_{\text {muog }}$ inferred from the gravimetric and muographic data respectively, are biased compared to the true averaged density $\bar{\rho}_{\text {true }}$ (Figs $11 \mathrm{c}-\mathrm{e}$ ). In practice, to bias the gravimetric and muographic data, we compute them using the synthetic density model (Fig. 3) shifted by a single constant. We study three cases: one corresponds to muography underestimating the density, which is likely when there is background contamination, another one corresponds to gravimetry overestimating the density and the last one corresponds to muography underestimating and gravimetry overestimating the density. In these three studied cases, we assume that the a priori density is equal to the true averaged density. When muography underestimates the density by $200 \mathrm{~kg} \mathrm{~m}^{-3}$ (Fig. 11c), the recovered densities are underestimated in the upper part of the dome which is adequately resolved by the muographic data, while the densities are overestimated in the bottom part of the model to compensate for the low upper density and to force the overall density model to be in accordance with the gravimetric data and the $a$ priori density. When the gravimetric data overestimate the density by $200 \mathrm{~kg} \mathrm{~m}^{-3}$ (Fig. 11d), the shallower density variations are not affected a lot as the muographic data also contribute to the resolution. But high densities are recovered in the bottom part of the model to explain the gravimetric data. The last case is a compromise between the two previous cases: muography underestimates the density by $100 \mathrm{~kg} \mathrm{~m}^{-3}$, gravimetry overestimates the density by $100 \mathrm{~kg} \mathrm{~m}^{-3}$ and the true and a priori densities lie in between (Fig. 11e). In this case, the top densities are underestimated while the bottom densities are overestimated for the same reasons as in the previous case. In terms of geometry of the recovered anomalies, the shapes of the structures are strongly altered (Figs $11 \mathrm{c}-\mathrm{e}$ ).

\section{DISCUSSION}

\subsection{Complementarity of gravimetry and muography}

The joint inversion of the gravimetric and muographic data significantly improves the resulting density models compared with the independent inversions, especially when using three muographic viewpoints (Figs 8 and 9). The final density model (Figs 8 and 9f) suitably reproduces the true density and gives the lowest averaged a posteriori density standard deviation $\left(57.4 \mathrm{~kg} \mathrm{~m}^{-3}\right)$. Note that the improvement of the joint inversion with respect to the independent inversions and the ability to recover the true density model strongly depends on the errors of the data. We used realistic data errors given the state-of-the-art of muography. However, future improvements in muography data acquisition and analysis are likely to decrease the expected errors, hence increase the contribution of the muographic data in the resolution of the density model. Jourde et al. (2015a) observed a gain in resolution below the volume sounded by muography when adding gravimetric data, but we do not observe any significant gain, possibly due to the different regularization used and the limited spatial extension of the gravimetric data with respect to the inverted volume. However, our results show that the gravimetric data overcome the lack of spatial coverage even when only one viewpoint is available in muography: gravimetry constrains the lateral variations while muography brings resolution at depth (Fig. 9d). When imaging a volcano, we are interested in both the shallow 
(a) A priori model underestimates the density by $200 \mathrm{~kg} / \mathrm{m}^{3}$
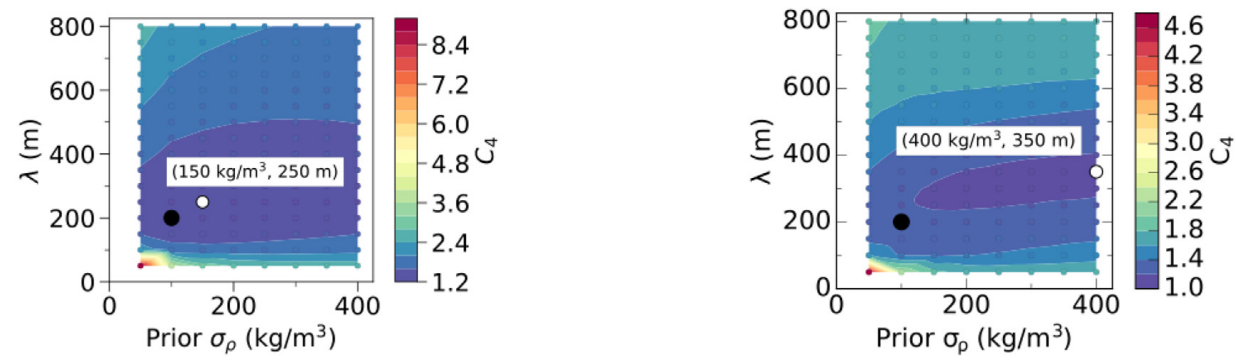

(b) A priori model overestimates the density by $200 \mathrm{~kg} / \mathrm{m}^{3}$
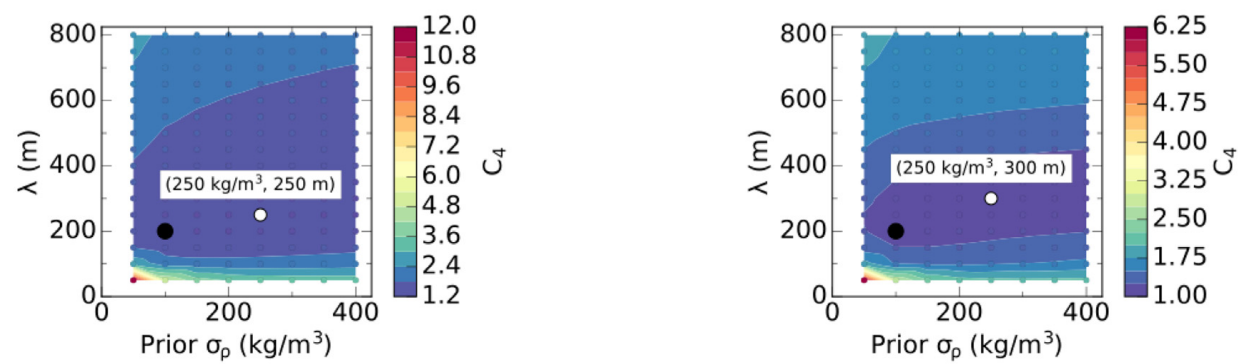

(c) Muography underestimates the density by $200 \mathrm{~kg} / \mathrm{m}^{3}$
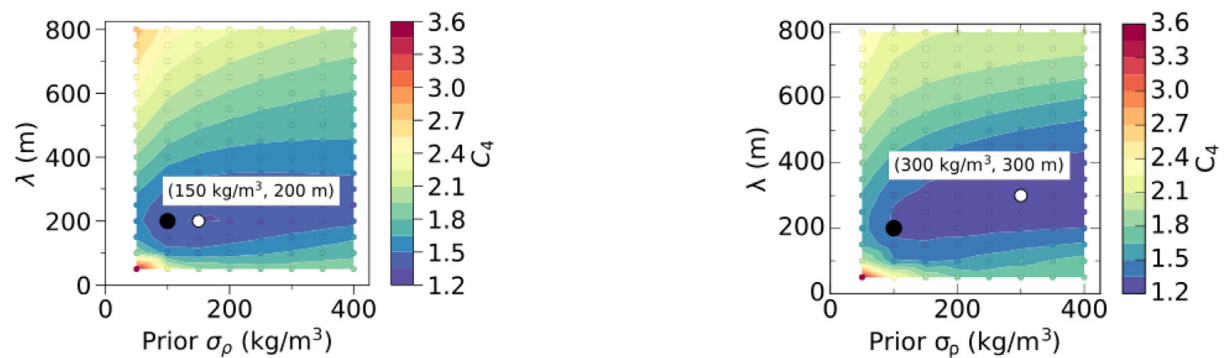

(d) Gravimetry overestimates the density by $200 \mathrm{~kg} / \mathrm{m}^{3}$
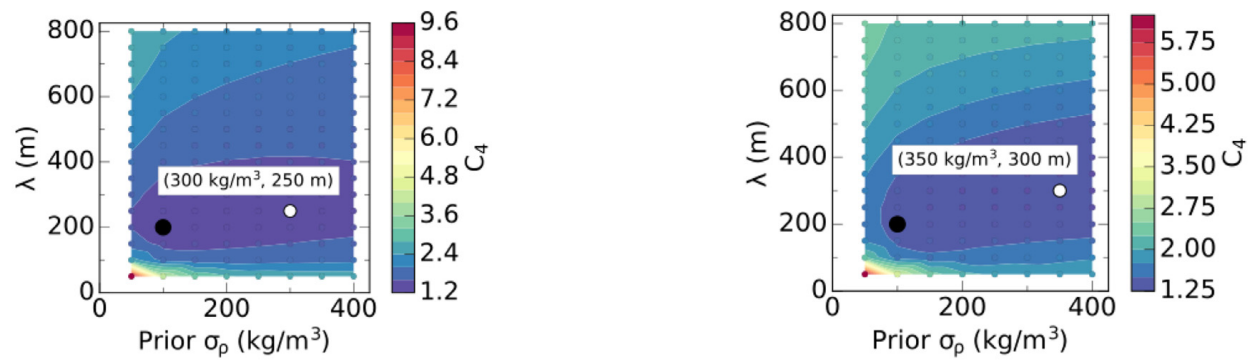

(e) Muography underestimates and gravimetry overestimates the density by $100 \mathrm{~kg} / \mathrm{m}^{3}$
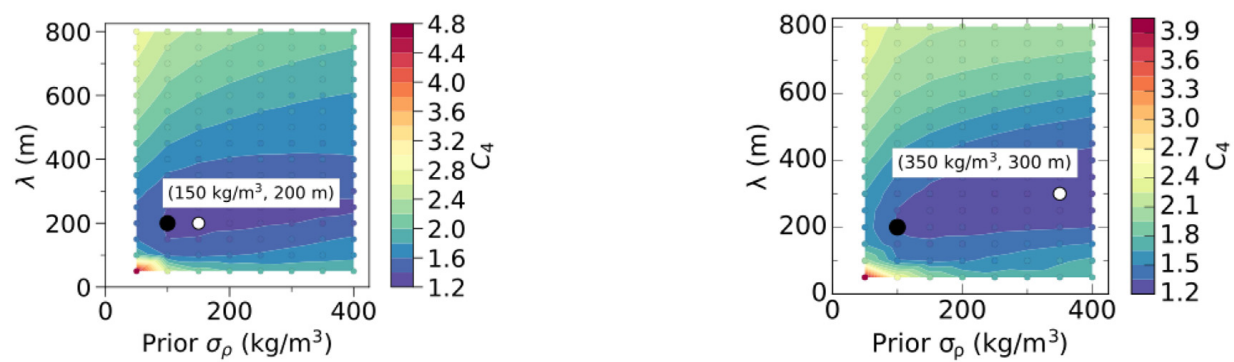

Figure 10. Fourfold CVSS criterion maps for the inversions with density shifts between the density inferred from gravimetric data $\bar{\rho}_{\text {grav }}$, the averaged density measured by muography $\bar{\rho}_{\text {muog }}$ and the a priori density $\bar{\rho}_{\text {prior }}$ with respect to the true averaged density $\bar{\rho}_{\text {true }}$. The white dots indicate the optimal values obtained from the fourfold CVSS criterion. The black dots indicate the reference model for $\left(\sigma_{\rho}, \lambda\right)=\left(100 \mathrm{~kg} \mathrm{~m}^{-3}, 200 \mathrm{~m}\right)$ (Figs 3 and 5). Left: joint inversion of the gravimetric data and the muographic data from the CDC viewpoint only. Right: joint inversion of the gravimetric data and the muographic data from the three viewpoints $\mathrm{CDC}, \mathrm{TDF}$ and $\mathrm{BDR}$. 
(a) A priori model underestimates the density by $200 \mathrm{~kg} / \mathrm{m}^{3}$
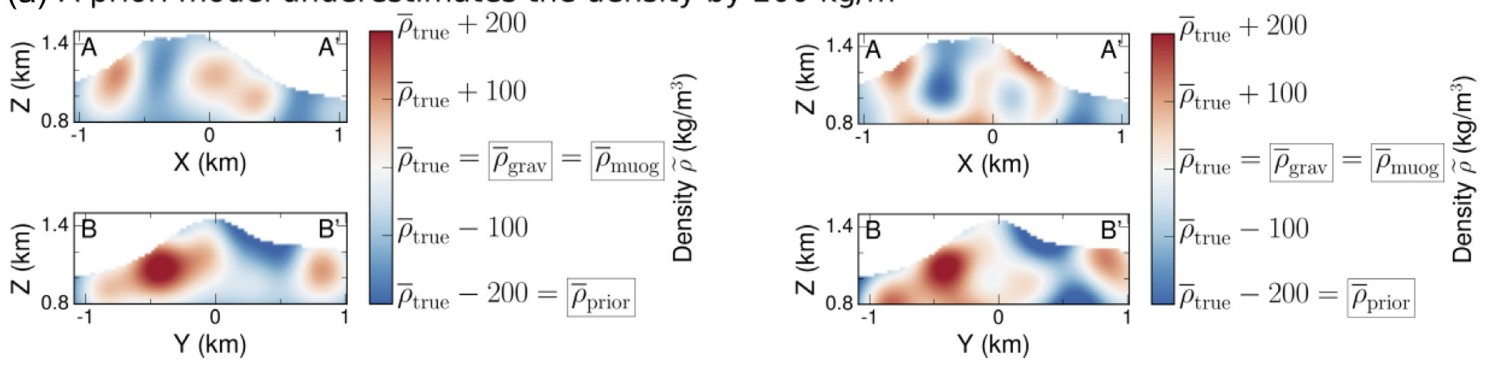

(b) A priori model overestimates the density by $200 \mathrm{~kg} / \mathrm{m}^{3}$
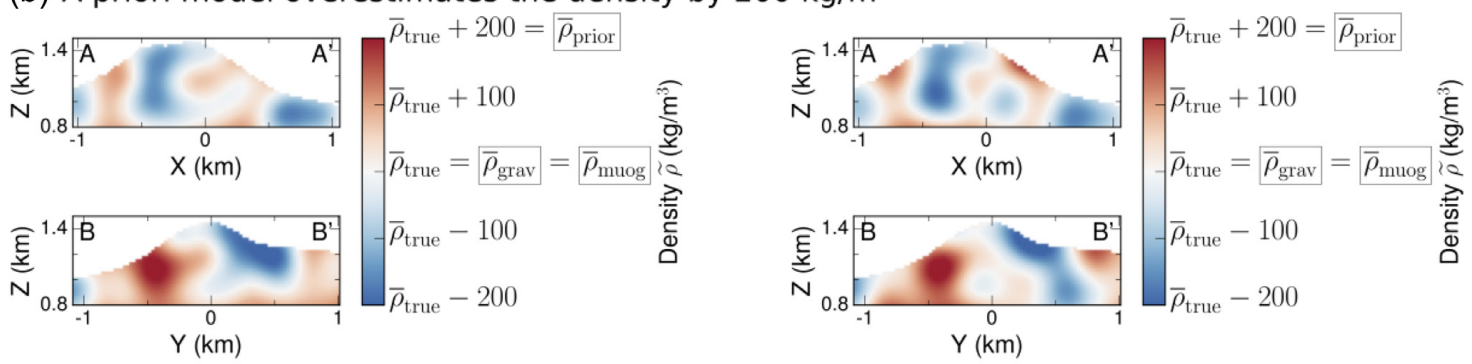

(c) Muography underestimates the density by $200 \mathrm{~kg} / \mathrm{m}^{3}$
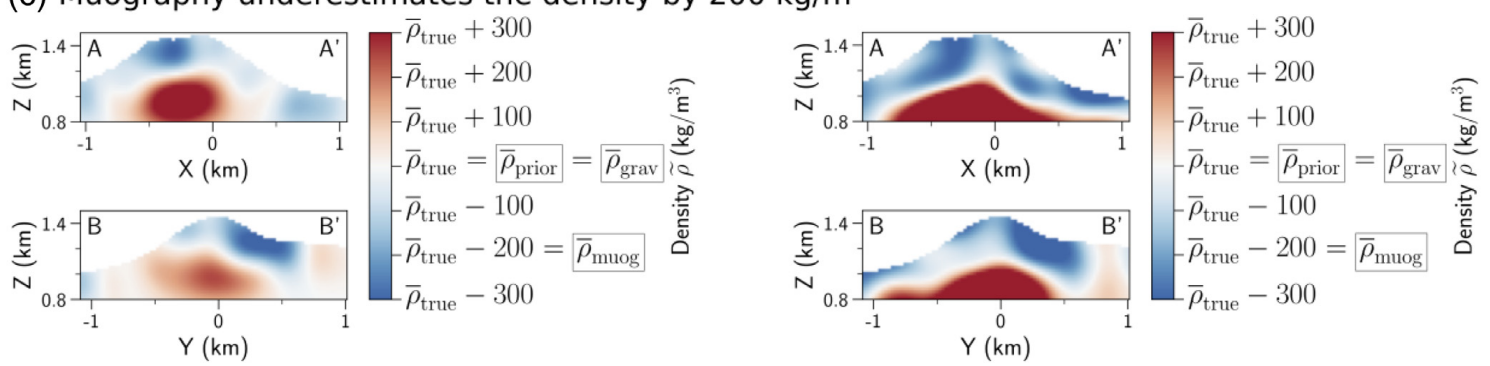

(d) Gravimetry overestimates the density by $200 \mathrm{~kg} / \mathrm{m}^{3}$
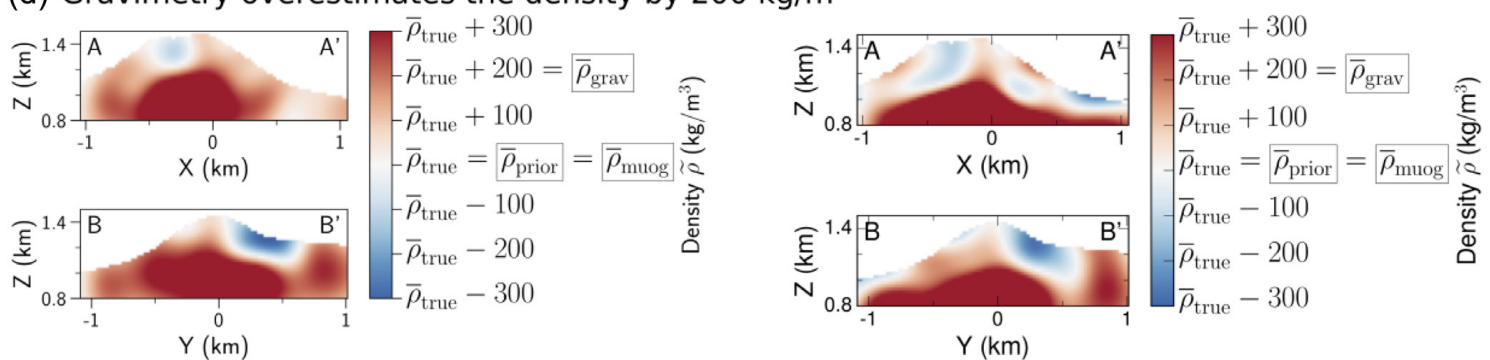

(e) Muography underestimates and gravimetry overestimates the density by $100 \mathrm{~kg} / \mathrm{m}^{3}$
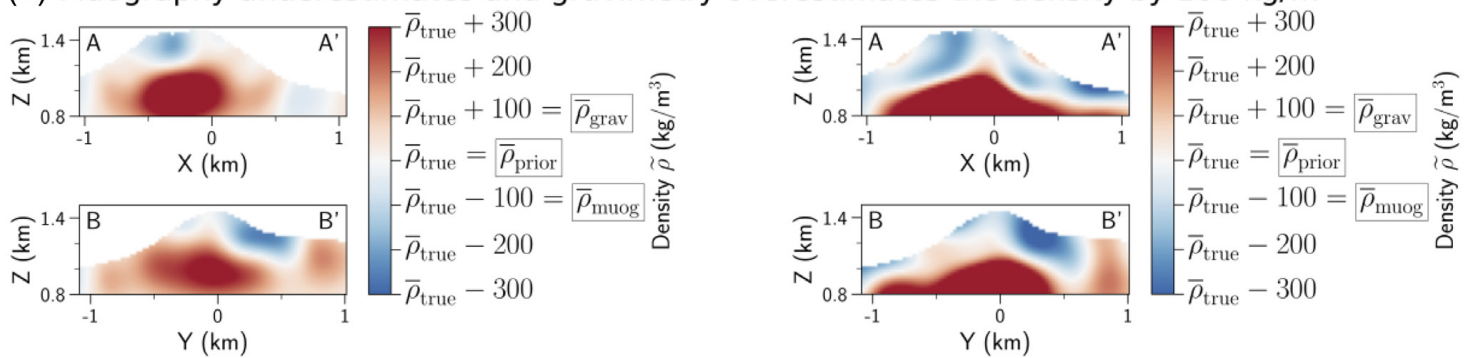

Figure 11. Effect of density shifts between the density inferred from gravimetric data $\bar{\rho}_{\text {grav }}$, the averaged density measured by muography $\bar{\rho}_{\text {muog }}$ and the $a$ priori density $\bar{\rho}_{\text {prior }}$ with respect to the true averaged density $\bar{\rho}_{\text {true }}$. The density models were obtained using the inversion hyperparameters determined with the fourfold CVSS criterion (white dots on Fig. 10). All colour bars are centred on $\bar{\rho}_{\text {true }}$. The range of the colour bars of panels (a) and (b) is the same as in the other figures of the paper $\left( \pm 200 \mathrm{~kg} \mathrm{~m}^{-3}\right)$, but the colour bars in panels (c)-(e) $\left( \pm 300 \mathrm{~kg} \mathrm{~m}^{-3}\right)$ are different. Left: joint inversion of the gravimetric data and the muographic data from the $\mathrm{CDC}$ viewpoint only. Right: joint inversion of the gravimetric data and the muographic data from the three viewpoints CDC, TDF and BDR. See Fig. 4 for the locations of the sections and of the muon detectors. 
structure and the structure at larger depth, so that we recommend using these two types of data jointly as previously established by Jourde et al. (2015a): muography improves the determination of the density structure at shallow elevations above the muons telescope, while gravimetry has the ability to resolve the density structure deeper than the muons telescope with a suitable network coverage.

\subsection{A priori density model and determination of associated hyperparameters}

The a priori density model to regularize the inversion was introduced using a Bayesian formalism. The a priori model is described as normally distributed densities with a Gaussian spatial correlation with a given correlation length as in eq. (9), resulting in smooth recovered density models. In previously published inversion of muographic data (e.g. Rosas-Carbajal et al. 2017), we could observe smearing out of the density anomalies along the muographic lines of sight. With our model regularization and three muographic viewpoints, these artefacts disappear (Figs 8e-f). This makes our approach particularly suitable for case studies with as few as three muon telescopes to image a kilometre scale volcano.

Another advantage of our regularization is that it avoids using weighting to counteract for the decreasing sensitivity. As shown by the sensitivities on Fig. 2, a given density node of the model is sensitive in different ways to the gravimetric and to the muographic data so that counteracting the sensitivity decay by weighting requires an arbitrary compromise between the two methods (e.g. RosasCarbajal et al. 2017). Imposing a spatial correlation to the model is a self-consistent approach in the sense that the a priori density model information does not depend on the data.

We consider an isotropic density smoothing using a unique constant isotropic correlation length. Cosburn et al. (2019) use both vertical and horizontal correlation lengths to account for the horizontal layering of the studied structures. In the case of a volcanic dome, such as the Puy de Dôme volcano, we expect spherical shapes rather than layered structures, therefore using an isotropic correlation length is appropriate.

In this study, we designed a synthetic model (Fig. 3) consistent with the assumption of a smooth Gaussian density distribution. However, the real Earth is likely to encompass anomalies at several scales and to present some discontinuities. Our approach is suitable for applications with little geological a priori knowledge or as a first step towards building a more complex model. In a second step, one could use the resulting smooth model as an initial model for nonlinear inversions looking for geometrical features like the precise location of interfaces between structures of presumed densities (e.g. Camacho et al. 2007; Cella et al. 2007; Lelièvre \& Farquharson 2013; Linde et al. 2014).

The same regularization approach was used by (Nishiyama et al. $2014 b$ ) for real data, with one muographic viewpoint. To the difference of the approach of Nishiyama et al. (2014b), where the hyperparameters were determined based on a given arbitrary synthetic model and then used for the real data inversion, our approach is generic as determination of the hyperparameters only relies on the data. For $k$-fold CVSS, usage shows that typical values for $k$ lie between 5 and 10 (e.g. James et al. 2013). We use a random distribution of the data to mix the gravimetric and the muographic data. In our case, a $k$-fold CVSS criterion with $k=4$ and the LOO methods are shown to give satisfying results. For such Bayesian joint inversion of gravimetric and muographic data, we therefore advocate using the LOO criterion or the CVSS criterion with $k \geq 4$.

\subsection{Dealing with biases}

In real data, the average density seen by muography is usually lower than the average density estimated with gravimetric data (e.g. Nishiyama et al. 2016; Rosas-Carbajal et al. 2017). Rosas-Carbajal et al. (2017) invert for a density model as well as a possible constant offset between the densities inferred by muographic data and gravimetric data. Lelièvre et al. (2019) investigate several methods to invert for a constant offset and suggest that the best approach is the automatic determination by least-squares minimization of a constant offset added to the observed muographic data.

The Bayesian approach presented in this paper has the advantage of taking into account the statistical errors on the data (variances and covariances), but it does not take into account any systematic errors. In Section 3.4, we show that biases in the a priori density, in the muographic data, or in the gravimetric data lead to density models that are likely to be misinterpreted.

When the mean a priori density is biased, the bias is difficult to detect in the resulting density model, but it affects only the parts of the model where the a posteriori density standard deviation is high thanks to the data resolving power (Figs 9d and f middle, and 11a and $b$ ). Where densities standard deviations are high, densities are close to the a priori. Therefore both the resulting densities and the associated a posteriori standard deviations should be considered for any geological interpretation of the resulting models.

Biases between the gravimetric and the muographic data sets are likely to alter not only the amplitudes of the recovered density anomalies, but also their shapes (Figs 11c-e), leading to misinterpretations. In particular, we obtain overestimated densities in the bottom part of the model to compensate either for muography underestimating densities either gravimetry overestimating densities, in accordance with the synthetic tests of Lelièvre et al. (2019). The occurrence of extreme densities in parts of the model badly resolved by the data could therefore be used as an indication of some shift between densities inferred from gravimetric and muographic data. This shift can be taken into account either automatically as shown in Lelièvre et al. (2019) or by hand performing several inversions with extreme values for the data to visualize the resulting range of possible density models. Gravimetric data can be shifted by adding the effect of a constant density offset in the model, computed using the forward formulation. Muographic data can easily be shifted (real data plus or minus the estimated bias). In real muographic data, the bias due to scattered low energy particles is likely not constant. It depends on the observation direction, on the topography shape and on the target surface structure, see for instance Gómez et al. (2017) for the effect of the muon incidence elevation angle, Niess et al. (2018b) for the effect of the topography or Cârloganu $\&$ the TOMUVOL collaboration (2018) for the effect of the local environment. In addition, uncertainties on the target composition result in non-uniform systematics as well. Detailed simulations can be used to estimate these effects. Reverse Monte-Carlo techniques are particularly efficient for this purpose (e.g. Niess et al. 2018a). The estimation of these biases would allow to get the possible range for the data. Hence, the range of resulting density models could be evaluated by inverting the data, plus and minus the biases estimates.

\section{CONCLUSION}

We show that the Bayesian formalism is well suited for the joint inversion of gravimetric and muographic data, even with a limited number of muon acquisition viewpoints. 
The inversion results outline the potential of joint muography and gravimetry inversions to retrieve the 3-D density distribution of geological structures such as volcanic edifices. In particular, the muographic data improve the resolution of structures at depth above the altitude of the muon detectors. They are able to constrain the vertical extension of structures, in contrast to gravimetric data which better constrain the lateral variations. When as few as one to three muographic viewpoints are available, gravimetric data bring complementary information at shallow depth and constrain density variations in the direction of the muography lines of sight, along which muography is blind, as well as away from the muon detectors. Below the muon detectors, gravimetry has the ability to resolve densities depending on the network coverage, with a resolving power rapidly decreasing with depth. We also determined that offsets between densities inferred by gravimetric and muographic data lead to recognizable artefacts in the retrieved densities, which should be taken into account in the inversions of real data.

The Bayesian formalism has the advantage to produce a model of densities with the associated a posteriori standard deviations that should be used to avoid misinterpretation of the resulting structures. We show that, for the joint inversion of gravimetric and muographic data, cross-validation criteria ( $k$-fold CVSS or LOO) are more robust criteria than the classically used L-Curves to select the inversion a priori hyperparameters for such joint inversion of gravimetric and muographic data. We also show that the regularization overcomes the artefacts due to the acquisition geometry: when three muographic viewpoints are used, we have no more smearing out along the muographic lines of sight as it can be observed in previously published studies. Therefore our method presents a significant improvement to the robustness of the inversions.

\section{ACKNOWLEDGEMENTS}

We thank the two reviewers for their comments that greatly helped improve the manuscript. We thank Niklas Linde, Nicolas Durrande and Rodolphe Le Riche for fruitful discussions. We also thank Olivier Coutant for the forward gravimetric computation code. Computations have been performed on the supercomputer facilities of the Mésocentre Clermont Auvergne. This research was financed by the French Government Laboratory of Excellence initiative no. ANR-10-LABX-0006, the Region Auvergne and the European Regional Development Fund. This is Laboratory of Excellence ClerVolc contribution number 321.

\section{REFERENCES}

Ambrosino, F. et al., 2015. Joint measurement of the atmospheric muon flux through the Puy de Dôme volcano with plastic scintillators and Resistive Plate Chambers detectors, J. geophys. Res., 120(11), 7290-7307.

Augier, A., 2011. Étude de l'éruption d'avril 2007 du Piton de la Fournaise (île de la Réunion) à partir de données d'interférométrie RADAR et GPS, développement et application de procédures de modélisation, Ph.D. thesis, Université Blaise Pascal, Clermont-Ferrand, France.

Barnoud, A., Coutant, O., Bouligand, C., Gunawan, H. \& Deroussi, S., 2016. 3-D linear inversion of gravity data: method and application to Basse-Terre volcanic island, Guadeloupe, Lesser Antilles, Geophys. J. Int., 205(1), 562-574.

Camacho, A.G., Montesinos, F.G. \& Vieira, R., 1997. A three-dimensional gravity inversion applied to São Miguel island (Azores), J. geophys. Res., 102(B4), 7717-7730.

Camacho, A.G., Nunes, J.C., Ortiz, E., Frana, Z. \& Vieira, R., 2007. Gravimetric determination of an intrusive complex under the Island of Faial
(Azores): some methodological improvements, Geophys. J. Int., 171, 478 494.

Cârloganu, C., the TOMUVOL collaboration, 2018. Density imaging of Puy de Dôme volcano with atmospheric muons in French Massif Central as a case study for volcano muography, in EGU General Assembly Conference Abstracts, Vol. 20, Vienna, Austria, p. 18420.

Cârloganu, C., Le Ménédeu, E. \&the TOMUVOL collaboration, 2016. Density imaging of Puy de Dôme volcano with atmospheric muons in French Massif Central as a case study for volcano muography, in EGU General Assembly Conference Abstracts, Vol. 18, p. 16681, Vienna Austria.

Cella, F., Fedi, M., Florio, G., Grimaldi, M. \& Rapolla, A., 2007. Shallow structure of the SommaVesuvius volcano from 3D inversion of gravity data, J. Volc. Geotherm. Res., 161, 303-317.

Cosburn, K., Roy, M., Guardincerri, E. \& Rowe, C., 2019. Joint inversion of gravity with cosmic ray muon data at a well-characterized site for shallow subsurface density prediction, Geophys. J. Int., 217(3), 1988-2002.

Coutant, O., Bernard, M.L., Beauducel, F., Nicollin, F., Bouin, M.P. \& Roussel, S., 2012. Joint inversion of P-wave velocity and density, application to La Soufrière of Guadeloupe hydrothermal system, Geophys. J. Int., 191(2), 723-742.

Fukuda, J. \& Johnson, K.M., 2008. A fully Bayesian inversion for spatial distribution of fault slip with objective smoothing, Bull. seism. Soc. Am., 98(3), 1128-1146.

Gentle, J.E., 2009. Computational Statistics, Springer Science \& Business Media.

Gómez, H. et al., 2017. Forward scattering effects on muon imaging, $J$. Instrum., 12(12), P12018.

Groom, D.E., Mokhov, N.V. \& Striganov, S.I., 2001. Muon stopping power and range tables $10-\mathrm{MeV}$ to $100-\mathrm{TeV}$, At. Data Nucl. Data Tables, 78, 183-356.

Harris, R.A. \& Segall, P., 1987. Detection of a locked zone at depth on the Parkfield, California, segment of the San Andreas fault, J. geophys. Res., 92(B8), 7945-7962.

James, G., Witten, D., Hastie, T. \& Tibshirani, R., 2013. An Introduction to Statistical Learning, Vol. 112, Springer.

Jourde, K., Gibert, D. \& Marteau, J., 2015a. Improvement of density models of geological structures by fusion of gravity data and cosmic muon radiographies, Geosci. Instrum., Methods Data Syst., 4(2), 177-188.

Jourde, K., Gibert, D., Marteau, J., de Bremond d'Ars, J. \& Komorowski, J.-C., 2015b. Muon dynamic radiography of density changes induced by hydrothermal activity at the La Soufrière of Guadeloupe volcano, Sci. Rep., 6, 33406.

Kusagaya, T. \& Tanaka, H.K.M., 2015. Development of the very long-range cosmic-ray muon radiographic imaging technique to explore the internal structure of an erupting volcano, Shinmoe-dake, Japan, Geosci. Instrum., Methods Data Syst., 4(2), 215-226.

Lechmann, A. et al., 2018. The effect of rock composition on muon tomography measurements, Solid Earth, 9(6), 1517-1533.

Lelièvre, P.G. \& Farquharson, C.G., 2013. Gradient and smoothness regularization operators for geophysical inversion on unstructured meshes, Geophys. J. Int., 195(1), 330-341.

Lelièvre, P.G., Barnoud, A., Niess, V., Cârloganu, C., Cayol, V. \& Farquharson, C.G., 2019. Joint inversion methods with relative density offset correction for muon tomography and gravity data, with application to volcano imaging, Geophys. J. Int., 218, 1685-1701.

Lesparre, N., Gibert, D. \& Marteau, J., 2012. Density muon radiography of La Soufrière of Guadeloupe volcano: Comparison with geological, electrical resistivity and gravity data, Geophys. J. Int., 185, 1-14.

Linde, N., Baron, L., Finizola, A., Revil, A., Muccini, F., Cocchi, L. \& Carmisciano, C., 2014. 3-D density structure and geological evolution of Stromboli volcano (Aeolian Islands, Italy) inferred from land-based and sea-surface gravity data, J. Volc. Geotherm. Res., 273, 58-69.

Nagahara, S. \& Miyamoto, S., 2018. Feasibility of three-dimensional density tomography using dozens of muon radiographies and filtered back projection for volcanos, Geosci. Instrum., Methods Data Syst., 7(4), 307316.

Nagamine, K., 2003. Introductory Muon Science, Cambridge Univ. Press. 
Nagamine, K., Iwasaki, M., Shimomura, K. \& Ishida, K., 1995. Method of probing inner-structure of geophysical substance with the horizontal cosmic-ray muons and possible application to volcanic eruption prediction, Nucl. Instrum. Methods Phys. Res., 356(2), 585-595.

Niess, V., Barnoud, A., Cârloganu, C. \& Le Ménédeu, E., 2018a. Backward Monte-Carlo applied to muon transport, Comput. Phys. Commun., 229, 54-67.

Niess, V., Barnoud, A., Cârloganu, C. \& Le Ménédeu, E., 2018b. Methods and tools for transmission muography, in EGU General Assembly Conference Abstracts, 20, 18110.

Nishiyama, R., Miyamoto, S. \& Naganawa, N., 2014a. Experimental study of source of background noise in muon radiography using emulsion film detectors, Geosci. Instrum., Methods Data Syst., 3, 29-39.

Nishiyama, R., Tanaka, Y., Okubo, S., Oshima, H., Tanaka, H.K.M. \& Maekawa, T., 2014b. Integrated processing of muon radiography and gravity anomaly data toward the realization of high-resolution 3-D density structural analysis of volcanoes: Case study of Showa-Shinzan lava dome, Usu, Japan, J. geophys. Res., 119(1), 699-710.

Nishiyama, R., Taketa, A., Miyamoto, S. \& Kasahara, K., 2016. Monte Carlo simulation for background study of geophysical inspection with cosmic-ray muons, Geophys. J. Int., 206, 1039-1050.

Nishiyama, R., Miyamoto, S., Okubo, S., Oshima, H. \& Maekawa, T., 2017. 3D density modeling with gravity and muon-radiographic observations in Showa-Shinzan lava dome, Usu, Japan, Pure appl. Geophys., 174(3), 1061-1070.

Oláh, L., Tanaka, H.K., Ohminato, T. \& Varga, D., 2018. High-definition and low-noise muography of the Sakurajima volcano with gaseous tracking detectors, Sci. Rep., 8(1), 3207.

Onizawa, S., Mikada, H., Watanabe, H. \& Sakashita, S., 2002. A method for simultaneous velocity and density inversion and its application to and exploration of subsurface structure beneath Izu-Oshima volcano and Japan, Earth Planets Space, 54, 803-817.

Portal, A., Gailler, L.-S., Labazuy, P. \& Lénat, J.-F., 2016. Geophysical imaging of the inner structure of a lava dome and its environment through gravimetry and magnetism, J. Volc. Geotherm. Res., 320, 88-99.

Rosas-Carbajal, M., Jourde, K., Marteau, J., Deroussi, S., Komorowski, J.-C. \& Gibert, D., 2017. Three-dimensional density structure of La Soufrière de Guadeloupe lava dome from simultaneous muon radiographies and gravity data, Geophys. Res. Lett., 44, 6743-6751.
Tanaka, H., Nagamine, K., Kawamura, N., Nakamura, S.N., Ishida, K. \& Shimomura, K., 2001. Development of the cosmic-ray muon detection system for probing internal-structure of a volcano, Hyperfine Interact., 138(1), 521-526.

Tanaka, H.K.M., Kusagaya, T. \& Shinohara, H., 2014. Radiographic visualization of magma dynamics in an erupting volcano, Nat. Commun., 5(3381), doi:10.1038/ncomms4381.

Tarantola, A., 2005. Inverse Problem Theory and Methods for Model Parameter Estimation, SIAM.

Tarantola, A. \& Valette, B., 1982. Inverse problems = quest for information, J. Geophys., 50, 159-170.

Wahba, G., 1990. Spline Models for Observational Data, Vol. 59, SIAM.

\section{APPENDIX: CONSTRUCTION OF THE GAUSSIAN RANDOM DENSITY MODEL}

A Gaussian random density model following the distribution defined by $\mathcal{N}\left(\bar{\rho}_{\text {true }}, \mathbf{C}_{\rho_{\text {true }}}\right)$ can be constructed from

$\rho_{\text {true }}=\bar{\rho}_{\text {true }}+\mathbf{L} X$,

where $X \sim \mathcal{N}(0, \mathbf{I})$ is a random vector drawn from a zero-mean normal distribution with an identity covariance matrix and $\mathbf{L}$ is the Cholesky decomposition of the covariance matrix $\mathbf{C}_{\rho_{\text {ruue }}}=\mathbf{L L}^{T}$ with a Gaussian correlation function of standard deviation $\sigma_{\rho_{\text {true }}}$ and of correlation length $\lambda_{\text {true }}$. Indeed, given the mean and variance of $X$

$$
\left\{\begin{array}{l}
\mathrm{E}[X]=0 \\
\mathrm{E}\left[X X^{T}\right]-\mathrm{E}[X] \mathrm{E}\left[X^{T}\right]=\mathbf{I},
\end{array}\right.
$$

the mean and variance of $\mathbf{L} X$ are (e.g. Gentle 2009):

$$
\begin{aligned}
& \begin{cases}\mathrm{E}[\mathbf{L} X]=\mathbf{L E}[X]=0 \\
\mathrm{E}\left[\mathbf{L} X(\mathbf{L} X)^{T}\right]-\mathrm{E}[\mathbf{L} X] \mathrm{E}\left[(\mathbf{L} X)^{T}\right] & =\mathrm{E}\left[\mathbf{L} X X^{T} \mathbf{L}\right]-\mathrm{E}[\mathbf{L} X] \mathrm{E}\left[X^{T} \mathbf{L}^{T}\right] \\
& =\mathbf{L}\left[\mathrm{E}\left[X X^{T}\right]-\mathrm{E}[X] \mathrm{E}\left[X^{T}\right]\right] \mathbf{L}^{T} \\
& =\mathbf{L} \mathbf{L}^{T}=\mathbf{C}_{\rho_{\text {true }}} .\end{cases} \\
& \text { Hence, } \quad \begin{array}{l}
\mathbf{L} X \sim \mathcal{N}\left(0, \mathbf{C}_{\rho_{\text {true }}}\right) \quad \text { and } \quad \rho_{\text {true }}=\bar{\rho}_{\text {true }}+\mathbf{L} X \sim \\
\mathcal{N}\left(\bar{\rho}_{\text {true }}, \mathbf{C}_{\rho_{\text {true }}}\right) .
\end{array}
\end{aligned}
$$

